\title{
THE SLICE MAP PROBLEM FOR $\sigma$-WEAKLY CLOSED SUBSPACES OF VON NEUMANN ALGEBRAS ${ }^{1}$
}

BY

JON KRAUS

\begin{abstract}
A $\sigma$-weakly closed subspace 5 of $B(\bar{H})$ is said to have Property $S_{\sigma}$ if for any $\sigma$-weakly closed subspace iT of a von Neumann algebra $, \pi,\{x \in \bar{\nabla}, \mathcal{Y}$ : $R_{\varphi}(x) \in \because$ for all $\left.\varphi \in B(\mathcal{K})_{*}\right\}=\$ \bar{\otimes}, \bar{T}$. where $R_{\varphi}$ is the right slice map associated with $\varphi$. It is shown that semidiscrete von Neumann algebras have Property $S_{\sigma}$, and various stability properties of the class of $\sigma$-weakly closed subspaces with Property $S_{o}$ are established. It is also shown that if $(\because \pi, G, \alpha)$ is a $W^{*}$-dynamical system such that $\because \pi$ has Property $S_{\sigma}$ and $G$ is compact abelian, then all of the spectral subspaces associated with $\alpha$ have Property $S_{\sigma}$. Some applications of these results to the study of tensor products of spectral subspaces and tensor products of reflexive algebras are given. In particular, it is shown that if $E_{1}$ is a commutative subspace lattice with totally atomic core, and $E_{2}$ is an arbitrary subspace lattice, then $\operatorname{alg}\left(E_{1} \otimes E_{2}\right)=$ $\operatorname{alg} L_{1} \bar{\otimes}_{\operatorname{alg}} E_{2}$.
\end{abstract}

Introduction. Slice maps, introduced by Tomiyama, have proved to be a powerful tool in studying tensor products of $C^{*}$-algebras and von Neumann algebras (see, e.g., $[23,24,26-28])$. In this paper we use slice maps to study tensor products of $\sigma$-weakly closed subspaces of von Neumann algebras, particularly tensor products of spectral subspaces, and tensor products of reflexive algebras.

If $\mathscr{X}$ and $\mathscr{X}$ are von Neumann algebras, then for each $\varphi$ in $\Re_{*}$, the predual of $\Re$, there is a unique $\sigma$-weakly continuous linear map $R_{\varphi}$ from $\Re \bar{\otimes} \Re$ to $\Re$ such that

$$
R_{\varphi}(a \otimes b)=\langle a, \varphi\rangle b \quad(a \in \Re, b \in \Re),
$$

called the right slice map associated with $\varphi$. Similarly, for each $\psi \in \Re_{*}$, there is a left slice map $L_{\psi}$ from $\Re \bar{\otimes} \Re$ to $\Re$. If $\mathcal{S} \subset \Re$ and $\mathcal{T} \subset \Re$ are $\sigma$-weakly closed subspaces, and $\delta \bar{\otimes} \mathcal{T}$ denotes the $\sigma$-weak closure of the algebraic tensor product of $\delta$ and $\mathcal{T}$, it is clear that if $x \in \mathcal{S} \bar{\otimes} \mathcal{T}$ then $R_{\varphi}(x) \in \mathcal{T}$ for all $\varphi \in \mathfrak{N}_{*}$. If

$$
\left\{x \in \delta \bar{\otimes} \Re: R_{\varphi}(x) \in \mathcal{T} \text { for all } \varphi \in \mathfrak{T}_{*}\right\}=\varsigma \bar{\otimes} \mathfrak{T}
$$

Received by the editors February 19, 1982 and, in revised form, July 20, 1982.

1980 Mathematics Subject Classification. Primary 46L10, 46L55, 46M05, 47D25.

Key words and phrases. Tensor product, slice map, Property $S_{\sigma}$, semidiscrete, $W^{*}$-dynamical system, spectral subspace, subspace lattice, reflexive algebra.

'This research was partially supported by NSF Grant MCS-8202332. 
for all pairs $(\tilde{T}, \mathcal{N})$, then we say $\delta$ has Property $S_{\sigma}$. (This is analogous to Wassermann's Property $S$ for $C^{*}$-algebras [26-28].) If every $\sigma$-weakly closed subspace of $B(\mathscr{H})$ has Property $S_{\sigma}$, then for any $\sigma$-weakly closed subspaces $\varsigma \subset B(\mathfrak{H})$ and $\mathcal{T} \subset B(\mathcal{H})$,

(*) $\left\{x \in B(\mathcal{H}) \bar{\otimes} B(\mathscr{K}): R_{\varphi}(x) \in \mathcal{T}\right.$ for all $\varphi \in B(\mathcal{H})_{*}$ and

$$
\left.L_{\psi}(x) \in \varsigma \text { for all } \psi \in B(\mathscr{K})_{*}\right\}=\varsigma \bar{\otimes} \bar{T} .
$$

Tomiyama has shown that $(*)$ is valid if $s$ and $T$ are von Neumann algebras [23, Theorem 2.1]. Tomiyama's result is equivalent to Tomita's commutation theorem for tensor products of von Neumann algebras, and so $(*)$ can be looked upon as an analogue of Tomita's theorem for $\sigma$-weakly closed subspaces. We show below that (*) is valid if either $\mathcal{H}$ or $\mathscr{K}$ is finite-dimensional, but the validity of $(*)$ in general remains an open problem.

In $\S 1$ we show that every semidiscrete von Neumann algebra has Property $S_{\sigma}$. This is analogous to Wassermann's (and T. Huruya's) result that every nuclear $C^{*}$-algebra has Property $S$ [28, Proposition 10]. We also show that $\ell\left(\mathbf{F}_{2}\right)$ (the regular group von Neumann algebra of the free group on two generators), a nonsemidiscrete von Neumann algebra, has Property $S_{\sigma}$. This can be compared with the result of De Cannière and Haagerup that the nonnuclear $C^{*}$-algebra $C_{r}^{*}\left(\mathbf{F}_{2}\right)$ (the reduced $C^{*}$-algebra of the free group on two generators) has Property $S[12$, Corollary to Theorem 2]. It remains an open question whether or not all von Neumann algebras have Property $S_{\sigma}$.

In $\S 2$ we use slice maps to study tensor products of spectral subspaces. We show that if $(\Re, G, \alpha)$ is a $W^{*}$-dynamical system such that $\Re$ has Property $S_{\sigma}$ and $G$ is compact (abelian), then for any subset $E$ of the dual group $\Gamma$ of $G$ the spectral subspace $\mathfrak{R}^{\alpha}(E)$ has Property $S_{\sigma}$. As an application of this result, we show that if $\left(\mathfrak{N}, G_{1}, \alpha\right)$ and $\left(\mathcal{N}, G_{2}, \beta\right)$ are $W^{*}$-dynamical systems, if $\mathscr{N}$ has Property $S_{\sigma}$, and if $G_{1}$ is compact, then

$$
(\mathscr{\Re} \bar{\otimes} \Re)^{\alpha \otimes \beta}\left(E_{1} \times E_{2}\right)=\mathscr{N}^{\alpha}\left(E_{1}\right) \bar{\otimes} \Re^{\beta}\left(E_{2}\right)
$$

for any subsets $E_{1} \subset \Gamma_{1}$ and $E_{2} \subset \Gamma_{2}$. We also prove that this equation is valid for arbitrary locally compact abelian groups $G_{1}$ and $G_{2}$, provided that either $\Re$ has Property $S_{\sigma}$ and $E_{1}$ is open, or both $E_{1}$ and $E_{2}$ are open.

In [15], the author established a connection between certain commutative subspace lattices and $W^{*}$-dynamical systems. In $\S 3$, using results from [15] and from $\S 2$, we show that if $\Re$ has Property $S_{\sigma}$, and if $\varrho_{1} \subset \Re$ is a commutative subspace lattice (CSL) whose core $\mathcal{L}_{1}^{\prime \prime}$ is totally atomic, then for any subspace lattice $\mathcal{L}_{2} \subset \mathcal{K}$, we have

$(* *) \quad \operatorname{alg}\left(\mathscr{L}_{1} \otimes \mathscr{L}_{2}\right) \cap(\Re \bar{\otimes} \Re)=\left[\left(\operatorname{alg} \mathscr{L}_{1}\right) \cap \Re\right] \bar{\otimes}\left[\left(\operatorname{alg} \mathscr{L}_{2}\right) \cap \Re\right]$,

where $\varrho_{1} \otimes L_{2}$ is the subspace lattice generated by $\varrho_{1}$ and $\varrho_{2}$. We also show that $(* *)$ holds if $\mathscr{L}_{1}$ is a chain, $\mathscr{L}_{2}$ is a finite width CSL, and $\Re$ and $\mathscr{L}_{1}^{\prime} \cap \Re$ have Property 
$S_{\sigma}$. This generalizes a result of Gilfeather, Hopenwasser and Larson, who proved that

$$
\operatorname{alg}\left(E_{1} \otimes E_{2}\right)=\left(\operatorname{alg} E_{1}\right) \bar{\otimes}\left(\operatorname{alg} E_{2}\right)
$$

if $E_{1}$ and $E_{2}$ are chains [10]. It should also be noted that if $E_{1}$ and $E_{2}$ are the projection lattices of von Neumann algebras $\mathfrak{N}$ and $\mathfrak{N}$, then $(* * *)$ becomes

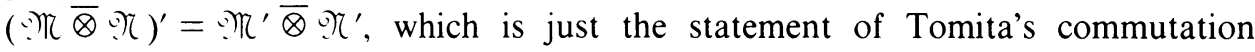
theorem.

The author would like to thank Jun Tomiyama for helpful comments on this paper. The author is also grateful to Jean De Cannière for providing a preliminary version of [8], and for several useful conversations about the material in [8].

1. Property $S_{\sigma}$. If $\mathscr{H}$ is a Hilbert space, let $B(\mathfrak{H})$ denote the algebra of bounded operators on $\mathcal{H}$. If $\mathcal{H}_{1}$ and $\mathscr{H}_{2}$ are Hilbert spaces, let $\mathcal{H}=\mathcal{H}_{1} \otimes \mathcal{H}_{2}$ be the Hilbert space tensor product of $\mathcal{H}_{1}$ and $\mathcal{H}_{2}$, and if $\mathcal{S}_{i} \subset B\left(\mathcal{H}_{i}\right)$ are $\sigma$-weakly closed (linear) subspaces, $i=1,2$, let $\delta_{1} \otimes \delta_{2}$ denote the $\sigma$-weakly closed linear span (in $B(\mathcal{H})$ ) of $\left\{s_{1} \otimes s_{2}: s_{i} \in \mathcal{S}_{i}\right\}$. In particular, if $\mathscr{N} \subset B\left(\mathcal{H}_{1}\right)$ and $\mathscr{T} \subset B\left(\mathcal{H}_{2}\right)$ are von Neumann algebras, then $\mathfrak{N} \bar{\otimes} \Re$ is the usual von Neumann algebra tensor product of $\Re$ and ) $\left(\right.$ (see, e.g., [21, Chapter 4]). If $\varphi \in \mathscr{N}_{*}$, then there is a unique $\sigma$-weakly continuous linear map $R_{\varphi}: \mathfrak{N} \bar{\otimes} \mathfrak{N} \rightarrow \Re$ such that

$$
\langle x, \varphi \otimes \psi\rangle=\left\langle R_{\varphi}(x), \psi\right\rangle \quad\left(x \in \Re \bar{\otimes} \Re, \psi \in \Re_{*}\right) .
$$

Similarly, for each $\psi \in \mathfrak{N}_{*}$, there is a unique $\sigma$-weakly continuous linear map $L_{\psi}$ : $\because \bar{\otimes}, \pi \rightarrow$ such that

$$
\langle x, \varphi \otimes \psi\rangle=\left\langle L_{\psi}(x), \varphi\right\rangle \quad\left(x \in \mathfrak{N} \bar{\otimes} \Re, \varphi \in \mathfrak{N}_{*}\right) .
$$

The maps $R_{\varphi}$ and $L_{\psi}$ are called right and left slice maps, and also satisfy (see, e.g. [23]):

$$
\begin{gathered}
\left\|R_{\varphi}\right\|=\|\varphi\|, \quad\left\|L_{\psi}\right\|=\|\psi\|, \\
R_{\varphi}(a \otimes b)=\langle a, \varphi\rangle b, \quad L_{\psi}(a \otimes b)=a\langle b, \psi\rangle \quad(a \in \Re, b \in \Re), \\
R_{\varphi}((1 \otimes c) x(1 \otimes d))=c R_{\varphi}(x) d \quad(c, d \in \Re, x \in \Re \bar{\otimes} \Re), \\
L_{\psi}((a \otimes 1) x(b \otimes 1))=a L_{\psi}(x) b \quad(a, b \in \Re, x \in \Re \bar{\otimes}), \\
R_{\varphi}(x)=0 \quad \text { for all } \varphi \in \Re_{*} \text { implies } x=0, \\
L_{\psi}(x)=0 \quad \text { for all } \psi \in \Re_{*} \text { implies } x=0 .
\end{gathered}
$$

DEFINITION 1.1. If $\mathcal{S} \subset \mathfrak{T}$ and $\mathcal{T} \subset \mathcal{K}$ are $\sigma$-weakly closed subspaces, let $F(\mathcal{S}, \mathcal{T})$ $=\left\{x \in \mathfrak{N} \bar{\otimes} \mathfrak{N}: R_{\varphi}(x) \in \mathcal{T}\right.$ and $L_{\psi}(x) \in \mathcal{S}$ for every $\varphi \in \mathfrak{N}_{*}$ and $\left.\psi \in \mathcal{N}_{*}\right\}$.

REMARK 1.2. The definition of $F(\delta, \mathcal{T})$ appears to depend on $\mathscr{\Re}$ and $\Re$ as well as $\delta$ and $\mathcal{T}$. However, this is not the case. For suppose $\mathscr{N} \subset B\left(\mathcal{H}_{1}\right)$ and $\mathscr{T} \subset B\left(\mathcal{H}_{2}\right)$. Let $\varphi \in \Re_{*}$, and let $\rho$ be any $\sigma$-weakly continuous extension of $\varphi$ to $B\left(\mathcal{H}_{1}\right)$. Then we can define $R_{\rho}: B\left(\mathcal{H}_{1}\right) \bar{\otimes} B\left(\mathcal{H}_{2}\right) \rightarrow B\left(\mathcal{H}_{2}\right)$, and it is clear from (1.2) that $R_{\varphi}=R_{\rho}$ on $\Re \bar{\otimes} \Re$. Hence if $x \in \mathfrak{N} \bar{\otimes} \Re$, then $R_{\varphi}(x) \in \mathcal{T}$ for all $\varphi \in \mathfrak{K}_{*}$ if and 
only if $R_{\rho}(x) \in \mathcal{T}$ for all $\rho \in B\left(\mathcal{H}_{1}\right)_{*}$. Moreover,

$$
\begin{aligned}
& \left\{x \in B\left(\mathcal{H}_{1}\right) \bar{\otimes}\left(\mathcal{H}_{2}\right): R_{\varphi}(x) \in \Re \text { and } L_{\psi}(x) \in \mathscr{N}\right. \\
& \left.\qquad \text { for all } \varphi \in B\left(\mathscr{H}_{1}\right)_{*} \text { and } \psi \in B\left(\mathcal{H}_{2}\right)_{*}\right\}=\Re \bar{\otimes} \Re
\end{aligned}
$$

[23, Theorem 2.1]. Thus, we also have

$$
\begin{aligned}
& F(\mathcal{S}, \mathcal{T})=\left\{x \in B\left(\mathcal{H}_{1}\right) \bar{\otimes} B\left(\mathcal{H}_{2}\right): R_{\varphi}(x) \in \mathcal{T} \text { and } L_{\psi}(x) \in \mathcal{S}\right. \\
& \left.\qquad \text { for all } \varphi \in B\left(\mathcal{H}_{1}\right)_{*} \text { and } \psi \in B\left(\mathcal{H}_{2}\right)_{*}\right\} .
\end{aligned}
$$

It follows easily from (1.2) that we always have

$$
\varsigma \bar{\otimes} \mathcal{T} \subset F(\delta, \mathcal{T}) \text {. }
$$

A natural question is: for which $\sigma$-weakly closed subspaces $\varsigma \subset \Re$ and $\Im \subset \Re$ do we have

$$
\varsigma \bar{\otimes} \mathcal{T}=F(\varsigma, \mathcal{T}) ?
$$

This question is of interest because a number of problems involving tensor products of $\sigma$-weakly closed subspaces can be reformulated in terms of (1.10). For example, if $\mathcal{S}_{1}, \mathcal{S}_{2} \subset \mathfrak{N}$ and $\mathscr{T}_{1}, \mathcal{T}_{2} \subset \mathfrak{K}$ are $\sigma$-weakly closed subspaces, it is easily checked that

$$
\left(\delta_{1} \cap \delta_{2}\right) \bar{\otimes}\left(\mathscr{T}_{1} \cap \mathcal{T}_{2}\right) \subseteq\left(\delta_{1} \bar{\otimes} \mathscr{T}_{1}\right) \cap\left(\delta_{2} \bar{\otimes} \mathcal{T}_{2}\right) \subseteq F\left(\delta_{1} \cap \Im_{2}, \mathcal{T}_{1} \cap \widetilde{T}_{2}\right) .
$$

Hence if (1.10) is always valid, then the intersection of tensor products of subspaces is always the tensor product of the intersections. If $1 \in \mathcal{S}$ and $1 \in \mathcal{T}$ (where we use 1 to denote the identity of both $\Re$ and $\Re)$, then it follows from (1.3)-(1.6) that

$$
(\varsigma \bar{\otimes} \sigma)^{\prime} \cap(\Re \bar{\Re} \Re)=F\left(\varsigma^{\prime} \cap \Re, \sigma^{\prime} \cap \Re\right)
$$

and we always have

$$
\left(\mathcal{S}^{\prime} \cap \Re\right) \bar{\Re}\left(\mathcal{T}^{\prime} \cap \mathcal{X}\right) \subseteq(\varsigma \bar{\otimes} \mathscr{T})^{\prime} \cap(\mathfrak{M} \bar{\otimes} \Re)
$$

so if (1.10) is always valid, then

$$
\left(\delta^{\prime} \cap \Re\right) \bar{\otimes}\left(\sigma^{\prime} \cap \Re\right)=(\delta \bar{\otimes} \sigma)^{\prime} \cap(\Re \bar{\otimes} \Re)
$$

whenever $1 \in \mathcal{S}$ and $1 \in \mathcal{T}$. Thus (1.7) follows from Tomita's commutation theorem and the double commutant theorem, and, conversely, Tomita's theorem follows from (1.7). (Note that (1.11) and (1.12) can both fail if $1 \notin \delta$. For example, let $\varsigma$ be the one-dimensional subspace generated by a nilpotent operator $s$ of index 2 in $B\left(\mathcal{H}_{1}\right)$ $\left(\operatorname{dim} \mathcal{H}_{1} \geqslant 2\right)$, and let $\mathcal{T}=B\left(\mathcal{H}_{2}\right)\left(\operatorname{dim} \mathcal{H}_{2} \geqslant 2\right)$. Then $F\left(\mathcal{S}^{\prime}, \mathcal{T}^{\prime}\right)=\mathcal{S}^{\prime} \otimes \mathbf{C} 1$, while $\delta \bar{\otimes} B\left(\mathcal{H}_{2}\right) \subset(\delta \bar{\otimes} \mathcal{T})^{\prime}$.) We will see other examples of the use of (1.10) in $\S 2^{2}$ and 3 below.

Definition 1.3. A von Neumann algebra $\Re$ is said to have Property $F_{\sigma}$ if (1.10) holds whenever $\mathcal{S}$ is a $\sigma$-weakly closed subspace of $\mathscr{T}$ and $\mathcal{T}$ is a $\sigma$-weakly closed subspace of a von Neumann algebra $\Re$.

The only von Neumann algebras known to have Property $F_{\sigma}$ are the finite-dimensional ones (Corollary 1.8 below). Note that by Remark 1.2, if $B(\mathcal{H})$ has Property $F_{\sigma}$ for every Hilbert space $\mathcal{K}$, then all von Neumann algebras have Property $F_{\sigma}$. However, deciding whether $B(\mathscr{H})$ has Property $F_{\sigma}$ appears to be a very difficult 
problem. It is equivalent to (1.10) always being valid, and Tomita's theorem, a deep result, is a special case of (1.10). Moreover, the proofs of Tomita's theorem all use selfadjointness in an essential fashion, and so appear to be of no use in studying the general validity of (1.10). We will consider the following, more tractable question: for which $\sigma$-weakly closed subspaces $\subseteq$ of $B(\mathcal{H})$ do we have

$$
\left\{x \in \varsigma \bar{\otimes} \mathfrak{K}: R_{\varphi}(x) \in \widetilde{T} \text { for all } \varphi \in B(\mathfrak{H})_{*}\right\}=\Sigma \bar{\otimes} \bar{T}
$$

for all pairs $(\sigma, \Re)$, where $\sigma$ is a $\sigma$-weakly closed subspace of a von Neumann algebra $\Re$ ?

Definition 1.4. A o-weakly closed subspace $\subseteq$ of $B(\mathcal{H})$ is said to have Property $S_{\sigma}$ if (1.13) holds whenever $T$ is a $\sigma$-weakly closed subspace of a von Neumann algebra or.

Remark 1.5. We will show below (Theorem 1.9) that $B(\mathcal{H})$ has Property $S_{\sigma}$ for any Hilbert space $\mathcal{H}$. Hence if $s \subset B\left(\mathcal{H}_{1}\right)$ and $\widetilde{T} \subset B\left(\mathcal{H}_{2}\right)$ are $\sigma$-weakly closed subspaces, $F(\delta, \mathcal{T}) \subseteq \varsigma \bar{\otimes} B\left(\mathcal{K}_{2}\right) \cap B\left(\mathcal{K}_{1}\right) \bar{\otimes}$. It follows that if $\delta$ has Property $S_{\sigma}$, then $F(\varsigma, T)=\varsigma \otimes \sigma$ whenever $T$ is a $\sigma$-weakly closed subspace of a von Neumann algebra $)$.

RemarK 1.6. Property $S_{\sigma}$ is modeled upon Wassermann's Property $S$ for $C^{*}$-algebras [26]. We recall the definition of Property $S$ for the convenience of the reader. If $A$ and $B$ are $C^{*}$-algebras, let $A \otimes B$ denote the minimal (spatial) $C^{*}$-tensor product of $A$ and $B$. If $\varphi \in A^{*}$, the right slice map $R_{\varphi}$ is the unique bounded linear map from $A \otimes B$ to $B$ satisfying

$$
\left\langle R_{\varphi}(x), \psi\right\rangle=\langle x, \varphi \otimes \psi\rangle \quad\left(x \in A \otimes B, \psi \in B^{*}\right) .
$$

The left slice maps $L_{\psi}, \psi \in B^{*}$, are similarly defined. These maps satisfy the exact analogues of (1.1)-(1.6) (where we require $A$ to be unital in (1.3) and $B$ to be unital in (1.4), and we replace $\Re_{*}$ by $A^{*}$ and $\Re_{*}$ by $B^{*}$ in (1.5) and (1.6)). A $C^{*}$-algebra $A$ is said to have Property $S$ if whenever $D$ is a $C^{*}$-subalgebra of a $C^{*}$-algebra $B$, we have

$$
\left\{x \in A \otimes B: R_{\varphi}(x) \in D \text { for all } \varphi \in A^{*}\right\}=A \otimes D,
$$

where $A \otimes D$ is canonically embedded in $A \otimes B$. Perhaps surprisingly, not all $C^{*}$-algebras have Property $S[\mathbf{2 7}, 28]$. In fact, if $\mathcal{H}$ is infinite-dimensional, then $B(\mathcal{H})$ does not have Property $S$ [27, Corollary 2.6]. There is a difference between Property $S$ and Property $S_{\sigma}: D$ is required to be a *-subalgebra of $B$, not just a norm closed subspace. If we required $\mathcal{T}$ to be a ${ }^{*}$-subalgebra in (1.13), then all von Neumann algebras would satisfy Property $S_{\sigma}$, by (1.7). However, it should be noted that nuclear $C^{*}$-algebras and $C_{r}^{*}\left(\mathbf{F}_{2}\right)$ satisfy the exact analogue of Property $S_{\sigma}$ : if $D \subset B$ is a norm closed subspace of a $C^{*}$-algebra $B$, and $A$ is nuclear or $A=C_{r}^{*}\left(\mathbf{F}_{2}\right)$, then

$$
\left\{x \in A \otimes B: R_{\varphi}(x) \in D \text { for all } \varphi \in A^{*}\right\}=A \otimes D,
$$

where $A \otimes D$ is the norm closed linear span of the elementary tensors $\{a \otimes d$ : $a \in A, d \in D\}$ in $A \otimes B$. (This follows easily from the proof of [28, Proposition 10] and [12, Corollary to Theorem 2].) It would be interesting to know whether Property $S$ always implies the exact analogue of Property $S_{\sigma}$. 
Our first result is simple, but crucial to what follows.

Proposition 1.7. Let $\S$ be a finite-dimensional subspace of $B(\mathcal{H})$. Then $\delta$ has Property $S_{\sigma}$.

Proof. Let $s_{1}, \ldots, s_{n}$ be a basis for $\delta$, and let $\Re$ be a von Neumann algebra. Since every finite-dimensional subspace of $B(\mathcal{H})$ is $\sigma$-weakly closed, we can choose $\varphi_{i} \in B(\mathcal{H})_{*}$ such that $\left\langle s_{i}, \varphi_{j}\right\rangle=\delta_{i j}, 1 \leqslant i, j \leqslant n$. If $x$ is in the algebraic tensor product of $\delta$ and $\Re$, it follows from (1.2) that

$$
x=\sum_{i=1}^{n} s_{i} \otimes R_{\varphi_{i}}(x) .
$$

But the maps $R_{\varphi_{1}}$ are $\sigma$-weakly continuous, so (1.14) is valid for all $x \in s \bar{\otimes} \cdot \pi$. Hence (1.13) holds for all $\sigma$-weakly closed subspaces $\mathcal{T} \subset \mathcal{N}$, and $S$ has Property $S_{\sigma}$.

COROLLARY 1.8. Let 9 be a finite-dimensional von Neumann algebra. Then $:$ has Property $F_{\sigma}$.

Proof. This is an immediate consequence of Proposition 1.7 and Remark 1.5.

Recall that a von Neumann algebra $\mathfrak{R}$ is semidiscrete [9] if the identity map on Th can be approximated in the topology of simple weak* convergence by normal morphisms of finite rank. (A morphism from a $C^{*}$-algebra $A$ with unit to a $C^{*}$-algebra $B$ with unit is a completely positive identity preserving linear map. We refer the reader to [9] for a detailed treatment of morphisms.) As noted in [9, p. 13], if $\mathfrak{N}$ is semidiscrete, then the identity map on $\mathfrak{K}_{*}$ can be approximated in the topology of simple norm convergence by morphisms of $\mathfrak{N}_{*}$ with finite rank, where a morphism of $\mathfrak{K}_{*}$ is a completely positive map from $\mathfrak{M}_{*}$ to itself which takes normal states to normal states. Using this fact, we can prove the following result.

THEOREM 1.9. Let 爪 be a semidiscrete von Neumann algebra. Then ? has Property $S_{\sigma}$

Proof. Let $\mathcal{T}$ be a $\sigma$-weakly closed subspace of a von Neumann algebra $(\eta)$, let $x \in \mathfrak{N} \bar{\otimes} \mathfrak{N}$, and suppose $R_{\varphi}(x) \in \mathcal{T}$ for all $\varphi \in \mathfrak{N}_{*}$. We want to show $\left.x \in \mathfrak{R}\right) \bar{\otimes}$ $\widetilde{T}$. Since the algebraic tensor product $\mathfrak{K}_{*} \otimes \Re_{*}$ is norm dense in $(\mathfrak{R} \bar{\otimes} \mathfrak{K})_{*}$, it suffices to show: if $\varepsilon>0, \varphi_{1}, \ldots, \varphi_{n} \in \mathfrak{R}_{*}$ and $\psi_{1}, \ldots, \psi_{n} \in \Re_{*}$, then there is a $y \in \mathfrak{N} \bar{\otimes} \mathcal{T}$ such that $\|y\| \leqslant\|x\|$, and $\sum_{i=1}^{n}\left|\left\langle x-y, \varphi_{i} \otimes \psi_{i}\right\rangle\right|<\varepsilon$. So let $\varepsilon>0$, and choose $\varphi_{1}, \ldots, \varphi_{n} \in \mathfrak{T}_{*}, \psi_{1}, \ldots, \psi_{n} \in \mathfrak{T}_{*}$. Let $K=n\|x\| \sup \left\{\left\|\psi_{1}\right\|, \ldots,\left\|\psi_{n}\right\|\right\}+1$. Since $\Re$ is semidiscrete, we can choose a finite rank morphism $\Phi$ of $\Re_{*}$ such that $\left\|\Phi\left(\varphi_{i}\right)-\varphi_{i}\right\|<\varepsilon / K, i=1, \ldots, n$. Then the adjoint $\Phi^{*}$ of $\Phi$ is a finite rank normal morphism of $\Re$. Hence there is a unique normal morphism $\Psi: \mathfrak{\pi} \bar{\otimes} \mathfrak{N} \rightarrow \mathfrak{\pi} \mathbb{\Re}$ such that $\Psi(a \otimes b)=\Phi^{*}(a) \otimes b(a \in \mathfrak{N}, b \in \Re)[9$, Lemma 2.5(ii)]. Let $y=\Psi(x)$. Then $\|y\| \leqslant\|x\|$, and $\left\langle R_{\varphi}(y), \psi\right\rangle=\langle y, \varphi \otimes \psi\rangle=\langle x, \Phi(\varphi) \otimes \psi\rangle=\left\langle R_{\Phi(\varphi)}(x), \psi\right\rangle$ 
for all $\varphi \in \mathscr{N}_{*}$ and $\psi \in \mathfrak{N}_{*}$. Thus $R_{\varphi}(y) \in \widetilde{T}$ for all $\varphi \in \mathcal{N}_{*}$. But $y \in \Phi^{*}(\mathfrak{N}) \bar{\otimes}$ $\Re$, and $\Phi^{*}(\mathfrak{T})$ is finite-dimensional, so $y \in \Phi^{*}(\mathcal{M}) \bar{\otimes} \widetilde{\top} \subseteq \Re \bar{\otimes} \widetilde{\mathcal{T}}$, by Proposition 1.7 and Remark 1.2. Finally,

$$
\begin{aligned}
\sum_{i=1}^{n}\left|\left\langle x-y, \varphi_{i} \otimes \psi_{i}\right\rangle\right| & =\sum_{i=1}^{n}\left|\left\langle x, \varphi_{i} \otimes \psi_{i}-\Phi\left(\varphi_{i}\right) \otimes \psi_{i}\right\rangle\right| \\
& \leqslant \sum_{i=1}^{n}\|x\|\left\|\varphi_{i}-\Phi\left(\varphi_{i}\right)\right\|\left\|\psi_{i}\right\|<\varepsilon
\end{aligned}
$$

as required.

Note that type I von Neumann algebras are semidiscrete [9. Proposition 3.5], and so have Property $S_{\sigma}$. It is a deep result of Connes [6] (for factors) and Choi and Effros [4] (the general case), that a separably acting von Neumann algebra is semidiscrete if and only if it is injective (as an object in the category of $C^{*}$-algebras with unit) if and only if it is approximately finite-dimensional (i.e. generated by an increasing sequence of finite-dimensional *-algebras). Moreover, $\mathfrak{R}$ is injective if and only if there is a projection of norm one from $B(\mathcal{H})$ onto $\mathfrak{N}$ [9, Theorem 5.3]. We refer the reader to [7] for an expository treatment of these and related results.

Proposition 1.10. Let $S \subset B(\mathcal{H})$ be a $\sigma$-weakly closed subspace having Property $S_{\sigma}$, and suppose e and $f$ are projections in $B(\mathcal{H})$ such that $e S f \subset \mathcal{S}$. Then $e \mathscr{S} f$ also has Property $S_{\sigma}$.

Proof. Since $e \varsigma f \subset \varsigma, e \delta f \bar{\otimes} \Re \subset \varsigma \bar{\otimes} \Re$ for any von Neumann algebra $\Re$. Hence if $\widetilde{T}$ is a $\sigma$-weakly closed subspace of $\Re, x \in e \delta f \bar{\otimes} \mathcal{K}$, and $R_{\varphi}(x) \in \mathcal{T}$ for all $\varphi \in B(\mathcal{H})_{*}$, then $x \in S \bar{\otimes}$. But $x=(e \otimes 1) x(f \otimes 1)$, so

$$
x \in(e \otimes 1)(\varsigma \otimes T)(f \otimes 1)=e \varsigma f \bar{\Phi} \mathcal{T} .
$$

Hence $e s f$ has Property $S_{\sigma}$.

Proposition 1.11. Let $\overline{\mathrm{S}} \subset \mathrm{B}(\mathcal{H})$ be a $\sigma$-weakly closed subspace, and let $\left\{\boldsymbol{e}_{i}\right\}_{i \in I}$ be a mutually orthogonal family of projections in $B(\mathcal{H})$ such that $\sum_{i \in I} e_{i}=1$ and $\mathcal{S} e_{i} \subset \mathcal{S}$ for all $i \in I$. If each $\mathrm{S}_{i}$ has Property $S_{\sigma}$, then $\mathcal{S}$ has Property $S_{\sigma}$.

Proof. Let $\mathcal{T}$ be a $\sigma$-weakly closed subspace of a von Neumann algebra $\Re$, let $x \in \mathcal{S} \bar{\otimes} \mathcal{X}$, and suppose $R_{\varphi}(x) \in \mathcal{T}$ for all $\varphi \in B(\mathcal{H})_{*}$. Then for each $i, x\left(e_{i} \bar{\otimes} 1\right)$ $\in \delta e_{i} \bar{\otimes} \mathcal{N}$, and $R_{\varphi}\left(x\left(e_{i} \otimes 1\right)\right)=R_{e_{i} \varphi}(x) \in \mathcal{T}$ for all $\varphi \in \mathcal{N}_{*}$ (where $\left\langle a, e_{i} \varphi\right\rangle=$ $\left.\left\langle a e_{i}, \varphi\right\rangle, a \in \mathfrak{N}, \varphi \in \Re_{*}\right)$. Hence $x\left(e_{i} \otimes 1\right) \in \delta e_{i} \bar{\otimes} \mathcal{T} \subset \delta \bar{\otimes} \sigma$ for all $i \in I$. But $\sum_{i \in I} e_{i} \otimes 1=1 \otimes 1$, so $x \in \mathcal{S} \overline{\mathcal{T}}$, as required.

It is easy to see that if $\mathscr{N}$ and $\Re$ are ${ }^{*}$-isomorphic von Neumann algebras, then $\mathfrak{N}$ has Property $S_{\sigma}$ if and only if $\mathscr{\Re}$ has Property $S_{\sigma}$. Combining this fact with Propositions 1.10 and 1.11 , we obtain

Proposition 1.12. If $\left\{\mathfrak{T R}_{i}\right\}_{i \in I}$ is any family of von Neumann algebras, then the direct sum $\oplus \Re_{i}$ has Property $S_{\sigma}$ if and only if each $\Re_{i}$ has Property $S_{\sigma}$. 
Proposition 1.12 is the analogue (with "is semidiscrete" replaced by "has Property $S_{\sigma}$ ") of [9, Proposition 3.1]. The next two results follow from Proposition 1.12 in exactly the same way [9, Corollaries 3.2 and 3.3] follow from [9, Proposition 3.1], and so the proofs are omitted.

COROLlaRY 1.13. If $\Re$ and $\Re$ are von Neumann algebras, and $\Phi: \Re \rightarrow \Re$ is a normal surjective homomorphism, then $\Re$ has Property $S_{\sigma}$ if and only if $\Re$ and $\operatorname{ker} \Phi$ have Property $S_{\sigma}$.

COROLlaRY 1.14. If $\mathfrak{T}$ is a von Neumann algebra, and $\left\{\pi_{i}\right\}_{i \in I}$ is a separating family of normal representations of $\Re$, then $\Re$ has Property $S_{\sigma}$ if and only if each of the algebras $\pi_{i}(\mathfrak{T})$ has Property $S_{\sigma}$.

Proposition 1.15. If $\varsigma_{1} \subset B\left(\mathcal{H}_{1}\right)$ and $\varsigma_{2} \subset B\left(\mathcal{H}_{2}\right)$ are nonzero $\sigma$-weakly closed subspaces, then $\varsigma_{1} \otimes \delta_{2}$ has Property $S_{\sigma}$ if and only if $\varsigma_{1}$ and $\varsigma_{2}$ have Property $S_{\sigma}$.

Proof. The proof of the "if" direction can be obtained by an obvious modification of the proof of [26, Lemma 14]. So assume $\varsigma_{1} \bar{\otimes} \varsigma_{2}$ has Property $S_{\sigma}$. Since $\varsigma_{2} \bar{\otimes} \varsigma_{1}$ is the image of $\varsigma_{1} \bar{\otimes} \varsigma_{2}$ under the natural *-isomorphism between $B\left(\mathcal{K}_{1}\right) \bar{\otimes}$ $B\left(\mathcal{H}_{2}\right)$ and $B\left(\mathcal{H}_{2}\right) \bar{\otimes} B\left(\mathcal{H}_{1}\right), \varsigma_{2} \otimes \varsigma_{1}$ also has Property $S_{\sigma}$. Hence it suffices to show that $\delta_{2}$ has Property $S_{\sigma}$. Let $\sigma_{1}$ be a $\sigma$-weakly closed subspace of a von Neumann algebra $\Re$, let $x \in S_{2} \bar{\otimes}$, and suppose $R_{\varphi}(x) \in \Im$ for all $\varphi \in B\left(\mathcal{K}_{2}\right)_{*}$. Choose $s \neq 0$ in $\delta_{1}$ and let $y=s \otimes x$. Then $R_{\psi \otimes \varphi}(y)=\langle s, \psi\rangle R_{\psi}(x) \in$ T for all $\psi \in B\left(: H_{1}\right)_{*}$ and $\varphi \in B\left(\mathcal{H}_{2}\right)_{*}$. Since the algebraic tensor product $B\left(\mathcal{H}_{1}\right)_{*} \otimes B\left(\mathcal{H}_{2}\right)_{*}$ is norm dense in $B\left(\mathcal{H}_{1} \otimes \mathcal{H}_{2}\right)_{*}$, it follows from (1.1) that

$$
R_{\rho}(y) \in \mathcal{T} \quad \text { for all } \rho \in B\left(\mathcal{H}_{1} \otimes \mathfrak{H}_{2}\right)_{*} \text {. }
$$

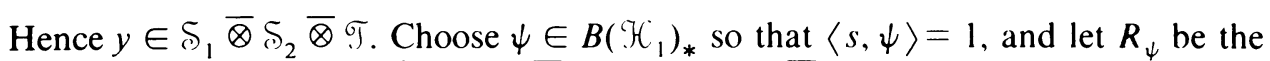
slice map from $B\left(\mathfrak{H}_{1}\right) \bar{\otimes} B\left(\mathfrak{H}_{2}\right) \bar{\otimes}$ to $B\left(\mathfrak{H}_{2}\right) \bar{\otimes} \mathfrak{X}$ associated with $\psi$. Then $x=R_{\psi}(y) \in \varsigma_{2} \otimes \tau_{T}$ as required, and so $\varsigma_{2}$ has Property $S_{\sigma}$.

Proposition 1.16. Let $\Re$ be a von Neumann algebra having Property $S_{\boldsymbol{a}}$. Then $M^{\prime}$ has Property $S_{\sigma}$.

Proof. Suppose first that $\mathscr{R} \subset B(\mathcal{H})$ is in standard form, so there is a conjugate linear isometry $j: \mathcal{H} \rightarrow \mathscr{H}$ such that $x \rightarrow j x j$ maps $\mathscr{N}$ onto $\mathscr{N}$ '. Let $\mathscr{T}$ be a $\sigma$-weakly closed subspace of a von Neumann algebra $\Re$, and suppose $x \in \mathfrak{N} \times \bar{\otimes}$, and $R_{\varphi}(x) \in \mathcal{T}$ for all $\varphi \in B(\mathcal{H})_{*}$. For $\varphi \in B(\mathcal{H})_{*}$, define $\varphi^{\prime} \in B(\mathcal{H})_{*}$ by $\left\langle a, \varphi^{\prime}\right\rangle=$ $\langle j a j, \varphi\rangle, a \in B(\mathcal{H})$. Let $y=(j \otimes 1) x(j \otimes 1)$, where $j \otimes 1$ has the obvious meaning. Then $y \in \Re \bar{R} \otimes \mathcal{K}$, and $R_{\varphi}(y)=R_{\varphi^{\prime}}(x) \in \mathcal{T}$ for all $\varphi \in B(\mathcal{H})_{*}$. Hence $y \in \mathscr{T} \bar{\otimes} \mathcal{T}$. But then $x=(j \otimes 1) y(j \otimes 1)$ is in $\mathfrak{K}^{\prime} \bar{\otimes} \mathcal{T}$, as required, so $\mathfrak{K}^{\prime}$ has Property $S_{\boldsymbol{\sigma}}$ if $\mathfrak{T}$ is in standard form. The proof of the general case is the exact analogue of the proof of the general case of [9, Proposition 3.7] (with "is semidiscrete" replaced by "has Property $S_{\sigma}$ " and [9, Propositions 3.4 and 3.6] replaced by Propositions 1.15 and 1.10), and so is omitted.

By results of Effros and Lance [9], the stability properties of the class of von Neumann algebras having Property $S_{\sigma}$ described in the preceding propositions and corollaries are also shared by the class of semidiscrete von Neumann algebras. 
However, the former class is strictly larger than the latter class. In particular, $\mathcal{R}\left(\mathbf{F}_{2}\right)$ (the regular group von Neumann algebra of the free group on two generators) is not semidiscrete [9, Theorem 5.11], but has Property $S_{\sigma}$. The proof that $R\left(\mathbf{F}_{2}\right)$ has Property $S_{\sigma}$ is based on work of De Cannière and Haagerup [8]. We first state a definition and a lemma from [8].

Let $A$ and $B$ be $C^{*}$-algebras, and let $M_{n}$ be the algebra of complex $n \times n$ matrices $(n=1,2, \ldots)$. If $\Phi$ is a linear map from $A$ to $B$, let $\Phi_{n}$ denote the linear map from $A \otimes M_{n}$ to $B \otimes M_{n}$ defined on elementary tensors by $\Phi_{n}(a \otimes b)=\Phi(a) \otimes b(a \in$ $A, b \in M_{n}$ ). The map $\Phi$ is said to be completely bounded if $\sup _{n}\left\|\Phi_{n}\right\|<\infty$. We write $\|\Phi\|_{C B}=\sup _{n}\left\|\Phi_{n}\right\|$ in this case.

Lemma 1.17 (J. De Cannière And U. HaAgerup [8]). Let $\Phi: \Re_{1} \rightarrow \Re_{2}$ be a $\sigma$-weakly continuous linear map between two von Neumann algebras $\mathfrak{R}_{1}$ and $\mathfrak{R}_{2}$. Then the following statements are equivalent:

(i) $\Phi$ is completely bounded.

(ii) For any Hilbert space $\mathscr{K}$, there is a (unique) $\sigma$-weakly continuous linear map $\Phi \otimes 1$ from $\mathfrak{M}_{1} \bar{\otimes} B(\mathfrak{K})$ to $\mathfrak{\Re}_{2} \bar{\otimes} B(\mathcal{K})$ satisfying

$$
(\Phi \otimes 1)(a \otimes b)=\Phi(a) \otimes b \quad\left(a \in \Re_{1}, b \in B\left(K_{K}\right)\right) .
$$

(iii) For any von Neumann algebra $\mathfrak{N}$, there is a (unique) $\sigma$-weakly continuous linear map $\Phi \otimes 1$ from $\Re_{1} \bar{\otimes} \Re$ to $\mathfrak{N}_{2} \bar{\otimes} \Re$ satisfying

$$
(\Phi \otimes 1)(a \otimes b)=\Phi(a) \otimes b \quad\left(a \in \mathfrak{K}_{1}, b \in \mathfrak{N}\right) .
$$

Moreover, if $\Phi$ is completely bounded, then the maps $\Phi \otimes 1$ obtained in (ii) and (iii) above are bounded in norm by $\|\Phi\|_{C B}$.

THEOREM 1.18. The group von Neumann algebra $R\left(\mathbf{F}_{2}\right)$ of the free group on two generators has Property $S_{\sigma}$.

Proof. Let $\Re=\mathscr{R}\left(\mathbf{F}_{2}\right)$. It is shown in [8] that the identity map on $\Re_{*}$ is the limit in the topology of simple norm convergence of a sequence $\left\{\Phi_{i}\right\}$ of finite rank linear maps from $\mathfrak{M}_{*}$ to $\mathfrak{M}_{*}$ satisfying $\left\|\Phi_{i}^{*}\right\|_{C B} \leqslant 1$, where $\Phi_{i}^{*}$ is the adjoint of $\Phi_{i}$ $(i=1,2, \ldots)$. Note that for each $i, \Phi_{i}^{*}$ is a finite rank $\sigma$-weakly continuous linear map from $\mathscr{N}$ to $\Re$. Moreover, by Lemma 1.17 , if $\Re$ is any von Neumann algebra there is, for each $i$, a unique $\sigma$-weakly continuous linear map $\Psi_{i}: \mathfrak{\Re} \bar{\otimes} \mathfrak{X} \rightarrow \mathfrak{\Re} \bar{\otimes}$ such that $\Psi_{i}(a \otimes b)=\Phi_{i}^{*}(a) \otimes b(a \in \mathfrak{K}, b \in \mathfrak{N})$ and $\left\|\Psi_{i}\right\| \leqslant 1$. Using these facts, the proof of Theorem 1.9 can be easily modified to show that $R\left(\mathbf{F}_{2}\right)$ has Property $S_{\sigma}$.

Our last result in this section will prove useful in $\$ 2$.

Proposition 1.19. Let $\subseteq \subset B(\mathcal{H})$ be a $\sigma$-weakly closed subspace having Property $S_{\sigma}$, let $\mathcal{S}_{1} \subset \mathcal{S}$ be a o-weakly closed subspace, and suppose there is a o-weakly continuous linear map $\Phi$ from $\mathcal{S}$ onto $\mathcal{S}_{1}$ such that $\Phi^{2}=\Phi$ and such that if $\mathscr{K}$ is any Hilbert space, there is a (unique) $\sigma$-weakly continuous linear map $\Phi \otimes 1$ from $\varsigma \bar{\otimes} B(\mathcal{K})$ to $\delta_{1} \bar{\otimes} B(\mathcal{K})$ satisfying

$$
(\Phi \otimes 1)(a \otimes b)=\Phi(a) \otimes b \quad(a \in \mathcal{S}, b \in B(\mathscr{K})) .
$$

Then $\mathcal{S}_{1}$ has Property $S_{\sigma}$. 
Proof. Let $\mathcal{T}$ be a $\sigma$-weakly closed subspace of a von Neumann algebra $\Re$. Since $\Phi \otimes 1: \varsigma \bar{\otimes} \rightarrow \delta_{1} \bar{\otimes} \mathfrak{N}$ is $\sigma$-weakly continous, $\Phi(\delta)=\varsigma_{1}$, and $(\Phi \otimes 1)^{2}=\Phi \otimes 1$, $(\Phi \otimes 1)(\delta \otimes \sigma)=\delta_{1} \bar{\otimes} \mathcal{T}$. Moreover, if $x \in \mathcal{S}_{1} \bar{\otimes} \mathfrak{K}$, then $(\Phi \otimes 1)(x)=x$. Hence

$$
(s \bar{\otimes}) \cap\left(s_{1} \bar{\otimes} \mathscr{N}\right)=s_{1} \bar{\otimes} \mathcal{T}
$$

Now let $x \in \mathcal{S}_{1} \bar{\otimes} \mathcal{K}$, and suppose $R_{\varphi}(x) \in \mathcal{T}$ for all $\varphi \in B(\mathcal{H})_{*}$. Since $\mathcal{S}$ has Property $S_{\sigma}, x \in \varsigma \bar{\otimes} \mathcal{T}$. Thus, by (1.15), $x \in \varsigma_{1} \bar{\otimes} \mathcal{T}$, and so $\varsigma_{1}$ has Property $S_{\sigma}$.

2. Applications to $W^{*}$-dynamical systems. A $W^{*}$-dynamical system $(\mathfrak{N}, G, \alpha)$ consists of a von Neumann algebra ग $\mathcal{N}$, a locally compact group $G$, and a homomorphism $\alpha$ of $G$ into the group of *automorphisms of $\mathcal{N}$ such that all of the maps $g \rightarrow\left\langle\alpha_{g}(x), \varphi\right\rangle$ are continuous $\left(x \in \mathcal{N}, \varphi \in \mathcal{N}_{*}\right)$. In the rest of this paper we will always assume that $G$ is abelian, for then there is a well-developed theory of spectral subspaces, which allows one to do harmonic analysis of the dynamical systems. (We refer the reader to $[\mathbf{1 , 3 , 5 , 1 7}$ or $\mathbf{1 8}]$ for detailed treatments of spectral subspace theory.)

If ( $\mathfrak{T}, G, \alpha)$ is a $W^{*}$-dynamical system, and $f \in L^{1}(G)$, we can define an operator $\alpha(f)$ on $\mathfrak{N}$ by

$$
\langle\alpha(f)(x), \varphi\rangle=\int_{G} f(g)\left\langle\alpha_{g}(x), \varphi\right\rangle d g \quad\left(x \in \mathfrak{R}, \varphi \in \mathfrak{M}_{*}\right)
$$

(where $d g$ is Haar measure). It can be checked that $\alpha(f)$ is a $\sigma$-weakly continuous bounded linear operator on $\Re$, that the action $(f, x) \rightarrow \alpha(f) x$ makes $\Re$ into an $L^{1}(G)$-module, and that $\|\alpha(f)(x)\| \leqslant\|f\|_{1}\|x\|$. If $E$ is a subset of the dual group $\Gamma$ of $G$, the spectral subspace $\mathfrak{T}^{\alpha}(E)$ is the $\sigma$-weakly closed linear span of $\{x \in \mathfrak{\pi}$ : $\left.\operatorname{sp}_{\alpha}(x) \subset E\right\}$, where

$$
\operatorname{sp}_{\alpha}(x)=\left\{\gamma \in \Gamma: \hat{f}(\gamma)=0 \text { for all } f \in L^{1}(G) \text { with } \alpha(f) x=0\right\} .
$$

Here $\hat{f}(\gamma)=\int_{G} f(g)(g, \gamma) d g$, where $g, \gamma \rightarrow(g, \gamma)$ is the dual pairing of $G$ and $\Gamma$. If $E$ is closed, then $\mathfrak{T}^{\alpha}(E)=\left\{x \in \mathfrak{N}: \operatorname{sp}_{\alpha}(x) \subset E\right\}$. If $\gamma \in \Gamma$, then $\mathfrak{N}^{\alpha}(\gamma) \equiv$ $\mathfrak{T}^{\alpha}(\{\gamma\})=\left\{x \in \mathfrak{N}: \alpha_{g}(x)=(g, \gamma) x\right.$ for all $\left.g \in G\right\}$. In particular, $\mathfrak{N}^{\alpha}(0)=\{x \in$ IR: $\alpha_{g}(x)=x$ for all $\left.g \in G\right\}$ is the fixed-point algebra of $\Re$ under $\alpha$.

Now suppose $\left(\mathfrak{N}, G_{1}, \alpha\right)$ and $\left(\Re, G_{2}, \beta\right)$ are $W^{*}$-dynamical systems. If $g_{i} \in G_{i}$, $i=1,2$, let $\alpha_{g_{1}} \otimes \beta_{g_{2}}$ be the unique *-automorphism of $\Re \bar{\otimes} \Re$ such that

$$
\left(\alpha_{g_{1}} \otimes \beta_{g_{2}}\right)(a \otimes b)=\alpha_{g_{1}}(a) \otimes \beta_{g_{2}}(b) \quad(a \in \mathfrak{N}, b \in \mathcal{N})
$$

[21, Corollary IV. 5.3]. It can be checked that ( $\left.\Re \bar{\otimes} \Re, G_{1} \times G_{2}, \alpha \otimes \beta\right)$ is again a $W^{*}$-dynamical system. Let $\Gamma_{i}$ denote the dual group of $G_{i}, i=1,2$. We can ask: if $E_{i} \subset \Gamma_{i}, i=1,2$, when do we have

$$
\Re^{\alpha \otimes \beta}\left(E_{1} \times E_{2}\right)=\Re^{\alpha}\left(E_{1}\right) \bar{\otimes} \Re^{\beta}\left(E_{2}\right),
$$

where $\Re=\Re \bar{\otimes} \Re$ ? Tomiyama proved [23, p. 14] that (2.1) holds if $E_{i}=\{0\}$, $i=1,2$, i.e., that the fixed-point algebra of the tensor product action is the tensor product of the fixed-point algebras. (This last statement makes sense, and is valid, for arbitrary locally compact groups.) The author proved that if $G_{1}$ and $G_{2}$ are 
compact, then (2.1) holds for arbitrary $E_{1}$ and $E_{2}$ [15, Theorem 4.3]. It was also noted in [15] that we always have

$$
\pi^{\alpha}\left(E_{1}\right) \otimes \pi^{\beta}\left(E_{2}\right) \subset \Re^{\alpha \otimes \beta}\left(E_{1} \times E_{2}\right) .
$$

Combining (2.2) with the next proposition allows us to use slice maps techniques in studying when $(2.1)$ is valid.

Proposition 2.1. Let $\left.(\because), G_{1}, \alpha\right)$ and $\left(\cdots, G_{2}, \beta\right)$ be $W^{*}$-dynamical systems, and let $(R=) \bar{\otimes} \otimes$. Then if $E_{i} \subset \Gamma_{i}, i=1,2$.

$$
\left(R^{\alpha \otimes \beta}\left(E_{1} \times E_{2}\right) \subset F\left(\because \pi^{\alpha}\left(E_{1}\right), \vartheta^{\beta}\left(E_{2}\right)\right) .\right.
$$

We have equality in (2.3) if the $E_{i}$ are closed.

Proof. Since $\left.F(\cdot)^{\alpha}\left(E_{1}\right), \Omega^{\beta}\left(E_{2}\right)\right)$ is a $\sigma$-weakly closed subspace of $(R$, to prove (2.3), it suffices to show that if $\operatorname{sp}_{\alpha \otimes \beta}(x) \subset E_{1} \times E_{2}$, then $x \in F\left(: \pi^{\alpha}\left(E_{1}\right), \mathcal{N}^{\beta}\left(E_{2}\right)\right)$. Moreover, by [5,2.1.3(g) and 2.1.4], we may assume $\operatorname{sp}_{\alpha \otimes \beta}(x)$ is compact. We will show that if $\operatorname{sp}_{\alpha \otimes \beta}(x)$ is a compact subset of $E_{1} \times E_{2}$, then $\operatorname{sp}_{\alpha}\left(L_{\psi}(x)\right) \subset E_{1}$ for all $\psi \in \mathfrak{N}_{*}$. The proof that $\operatorname{sp}_{\beta}\left(R_{\varphi}(x)\right) \subset E_{2}, \varphi \in \Re_{*}$, is similar. Let $\gamma \in \Gamma_{1}, \gamma \notin E_{1}$. We will show that there is an $f_{1} \in L^{1}\left(G_{1}\right)$ such that $\hat{f}_{1}(\gamma)=1$ and $\alpha\left(f_{1}\right)\left(L_{\psi}(x)\right)=0$ for all $\psi \in \mathfrak{N}_{*}$. This implies that $\gamma \notin \operatorname{sp}_{\alpha}\left(L_{\psi}(x)\right), \psi \in \mathfrak{N}_{*}$, as desired. Let $K=$ $\operatorname{sp}_{\alpha \otimes \beta}(x)$. Since $K$ is compact, the projection of $K$ on $\Gamma_{1}$ is closed, and so we can choose an open neighborhood $U$ of $\gamma$ such that $\left(\bar{U} \times \Gamma_{2}\right) \cap K=\varnothing$. By the regularity of $L^{1}\left(G_{1}\right)[19,2.6 .2]$, we can choose $f_{1} \in L^{\prime}\left(G_{1}\right)$ such that $\hat{f}_{1}(\gamma)=1$ and $\operatorname{supp} \hat{f}_{1} \subset U$. If $f_{2} \in L^{1}\left(G_{2}\right)$ is arbitrary, we can define $f_{1} \otimes f_{2} \in L^{1}\left(G_{1} \times G_{2}\right)$ by $\left(f_{1} \otimes f_{2}\right)\left(g_{1}, g_{2}\right)$ $=f_{1}\left(g_{1}\right) f_{2}\left(g_{2}\right)\left(g_{i} \in G_{i}\right)$. Then $\left(f_{1} \otimes f_{2}\right)$ vanishes on a neighborhood of $K$, and so by a Tauberian Theorem $[19,7.2 .5(\mathrm{a})]$,

$$
(\alpha \otimes \beta)\left(f_{1} \otimes f_{2}\right)(x)=0, \quad f_{2} \in L^{1}\left(G_{2}\right) .
$$

It is easily checked that

$$
(\alpha \otimes \beta)\left(f_{1} \otimes f_{2}\right)(a \otimes b)=\alpha\left(f_{1}\right)(a) \otimes \beta\left(f_{2}\right)(b) \quad(a \in \mathfrak{N}, b \in \Re) .
$$

Hence, if $\varphi \in \mathscr{N}_{*}$ and $\psi \in \mathscr{X}_{*}$,

$$
\begin{aligned}
0 & =\left\langle(\alpha \otimes \beta)\left(f_{1} \otimes f_{2}\right)(x), \varphi \otimes \psi\right\rangle=\left\langle x, \varphi \circ \alpha\left(f_{1}\right) \otimes \psi \circ \beta\left(f_{2}\right)\right\rangle \\
& =\left\langle\alpha\left(f_{1}\right)\left(L_{\psi \circ \beta\left(f_{2}\right)}(x)\right), \varphi\right\rangle,
\end{aligned}
$$

so

$$
\alpha\left(f_{1}\right)\left(L_{\psi \circ \beta\left(f_{2}\right)}(x)\right)=0 \quad\left(f_{2} \in L^{1}\left(G_{2}\right), \psi \in \mathscr{T}_{*}\right) .
$$

But $\left\{\psi \circ \beta(f): f \in L^{1}\left(G_{2}\right), \psi \in \mathcal{N}_{*}\right\}$ is norm dense in $\mathcal{K}_{*}[5,2.1 .3(\mathrm{j})]$, and so it follows from (2.4) and (1.1) that $\alpha\left(f_{1}\right)\left(L_{\psi}(x)\right)=0$ for all $\psi \in \mathcal{T}^{*}$, as desired.

Next suppose $E_{1}$ and $E_{2}$ are closed. Then

$$
\Re^{\alpha \otimes \beta}\left(E_{1} \times E_{2}\right)=\left\{x \in \Re:(\alpha \otimes \beta)(f)(x)=0 \text { for all } f \in I_{0}\left(E_{1} \times E_{2}\right)\right\}
$$

$\left[1\right.$, p. 225], where $I_{0}\left(E_{1} \times E_{2}\right)=\left\{f \in L^{1}\left(G_{1} \times G_{2}\right): \hat{f}\right.$ vanishes on a neighborhood of $\left.E_{1} \times E_{2}\right\}$. Let $J$ be the norm closed subspace of $L^{1}\left(G_{1} \times G_{2}\right)$ generated by $\left\{f_{1} \otimes f_{2}\right.$ : either $f_{1} \in I_{0}\left(E_{1}\right)$ or $\left.f_{2} \in I_{0}\left(E_{2}\right)\right\}$. Then since $I_{0}\left(E_{i}\right)$ is an ideal, $i=1,2$, and the 
norm closed linear span of $\left\{f_{1} \otimes f_{2}: f_{i} \in L^{1}\left(G_{i}\right)\right\}$ is $L^{1}\left(G_{1} \times G_{2}\right)$ [11, Corollary 4, p. 61 ], $J$ is an ideal. Moreover, it follows easily from the regularity of $L^{1}\left(G_{1}\right)$ and $L^{1}\left(G_{2}\right)$ that $Z(J) \equiv\{\gamma \in \Gamma: \hat{f}(\gamma)=0$ for all $f \in J\}=E_{1} \times E_{2}$, and hence $I_{0}\left(E_{1} \times E_{2}\right) \subset J[19,7.2 .5(\mathrm{a})]$. So to prove equality holds in (2.3), it suffices to show: if $x \in F\left(\Re^{\alpha}\left(E_{1}\right), \Re^{\beta}\left(E_{2}\right)\right)$, then

$$
(\alpha \otimes \beta)\left(f_{1} \otimes f_{2}\right)(x)=0,
$$

if either $f_{1} \in I_{0}\left(E_{1}\right)$ or $f_{2} \in I_{0}\left(E_{2}\right)$. So assume $f_{1} \in I_{0}\left(E_{1}\right)$ and $f_{2} \in L^{1}\left(G_{2}\right)$, and $x \in F\left(\mathcal{N}^{\alpha}\left(E_{1}\right), \mathscr{\Re}^{\beta}\left(E_{2}\right)\right)$. Then if $\psi \in \Re_{*}, L_{\psi \circ \beta\left(f_{2}\right)}(x) \in \mathscr{N}^{\alpha}\left(E_{1}\right)$, so $\alpha\left(f_{1}\right)\left(L_{\psi \circ \beta\left(f_{2}\right)}(x)\right)=0$. But for $\psi \in \Re_{*}$,

$$
L_{\psi}\left((\alpha \otimes \beta)\left(f_{1} \otimes f_{2}\right)(x)\right)=\alpha\left(f_{1}\right)\left(L_{\psi \circ \beta\left(f_{2}\right)}(x)\right),
$$

so (2.5) holds by (1.6). A similar argument shows that (2.5) holds if $f_{1} \in L^{1}\left(G_{1}\right)$ and $f_{2} \in I_{0}\left(E_{2}\right)$. This completes the proof.

If $G$ is compact, the spectral subspace structure of a $W^{*}$-dynamical system $(\mathfrak{N}, G, \alpha)$ is particularly simple: there are $\sigma$-weakly continuous projections from : ) onto the spectral eigenspaces $\Re^{\alpha}(\gamma)$ of $\Re$, and $\Re$ is the $\sigma$-weakly closed linear span of its spectral eigenspaces. In fact, if we set $e_{\gamma}(g)=(\overline{g, \gamma})(g \in G, \gamma \in \Gamma)$, and $p_{\gamma}=\alpha\left(e_{\gamma}\right)$, then $p_{\gamma}(\mathfrak{T})=\mathfrak{I}^{\alpha}(\gamma), p_{\gamma}^{2}=p_{\gamma}$, and $p_{\gamma} p_{\lambda}=0$ if $\gamma \neq \lambda$. Moreover, if $E$ is any subset of $\Gamma$, then

$$
\Re^{\alpha}(E)=\underset{\gamma \in l}{\bigvee} \mathscr{M}^{\alpha}(\gamma)
$$

where $\vee$ denotes the $\sigma$-weakly closed linear span.

TheOREM 2.2. Let $(\mathfrak{M}, G, \alpha)$ be a $W^{*}$-dynamical system, and suppose that $G$ is compact and $\mathfrak{N}$ has Property $S_{\sigma}$. Then $\mathfrak{N}^{\alpha}(E)$ has Property $S_{\sigma}$ for any $E \subset \mathrm{I}$.

Proof. Let $\gamma \in \Gamma$. We will first show $\mathfrak{S}^{\alpha} \mathbb{R}^{\alpha}(\gamma)$ has Property $S_{\sigma}$. Let $\Phi=p_{\gamma}$. Then $\Phi$ is a $\sigma$-weakly continuous linear map from $\mathfrak{N}$ onto $\mathfrak{N}^{\alpha}(\gamma)$ such that $\Phi^{2}=\Phi$. Moreover, if $\mathscr{K}$ is any Hilbert space, we can define a $W^{*}$-dynamical system $(\Re \bar{\otimes} B(\mathscr{K}), G, \beta)$ by setting

$$
\beta_{g}=\alpha_{g} \otimes 1, \quad g \in G,
$$

where 1 is the identity automorphism of $B(\mathscr{K})$. Then a calculation shows that $\beta\left(e_{\gamma}\right)$ is a $\sigma$-weakly continuous linear map from $\Re \bar{\otimes} B(\mathscr{K})$ to $\Re^{\alpha}(\gamma) \bar{\otimes} B(\mathscr{K})$ satisfying

$$
\beta\left(e_{\gamma}\right)(a \otimes b)=\Phi(a) \otimes b \quad(a \in \mathfrak{N}, b \in B(\mathscr{K})) .
$$

Hence $\Re^{\alpha}(\gamma)$ has Property $S_{\sigma}$ by Proposition 1.19.

Next let $E \subset \Gamma$, let $\mathcal{T}$ be a $\sigma$-weakly closed subspace of a von Neumann algebra $\Re$, and suppose $x \in \Re^{\alpha}(E) \bar{\otimes} \Re$ and $R_{\varphi}(x) \in \mathcal{T}$ for all $\varphi \in \Re_{*}$. Define a $W^{*}$-dynamical system $(\mathfrak{N} \bar{\otimes} \Re, G, \beta)$ by means of $(2.7)$ (where 1 is the identity automorphism of $\mathcal{R}$ ). For $\gamma \in \Gamma$, set $x_{\gamma}=\beta\left(e_{\gamma}\right)(x)$. Then it follows from (2.8) that $x_{\gamma} \in \Re^{\alpha}(\gamma) \bar{\otimes} \Re$, and $R_{\varphi}\left(x_{\gamma}\right)=R_{\varphi \circ p_{\gamma}}(x) \in \widetilde{T}$ for all $\varphi \in \Re_{*}$. But $\Re^{\alpha}(\gamma)$ has Property $S_{\sigma}$, so $x_{\gamma} \in \Re^{\alpha}(\gamma) \bar{\otimes} \mathcal{T}, \gamma \in \Gamma$. If $\gamma \notin E$, then $p_{\gamma}\left(\Re^{\alpha}(E)\right)=0$, so $x_{\gamma}=0$, while if $\gamma \in E$, then $\mathfrak{T}^{\alpha}(\gamma) \subseteq \mathfrak{T}^{\alpha}(E)$. Hence $x_{\gamma} \in \mathfrak{N}^{\alpha}(E) \bar{\otimes} \mathcal{G}$ for all $\gamma \in \Gamma$. By 
$[5,2.1 .4], x$ is in the $\sigma$-weak closure of $\left\{\beta(f)(x): f \in L^{1}(G),\|f\|_{1} \leqslant 1\right.$ and $\hat{f}$ has compact support $\}$. But if $\hat{f}$ has compact support, it has finite support, and so $\beta(f)(x)=\beta\left(\Sigma_{\gamma \in \Gamma} \hat{f}(\gamma) e_{\gamma}\right)(x)=\Sigma_{\gamma \in \Gamma} \hat{f}(\gamma) x_{\gamma} \in \mathscr{N}^{\alpha}(E) \bar{\otimes} \mathcal{T}$. Hence $x \in \mathfrak{T}^{\alpha}(E)$ $\bar{\otimes} \mathcal{T}$, and so $M^{\alpha}(E)$ has Property $S_{\sigma}$.

RemarK 2.3. Suppose $(\mathfrak{N}, G, \alpha)$ is a $W^{*}$-dynamical system, and suppose $G$ is compact. Let $E$ be a subset of $\Gamma$. It follows from a straightforward modification of the proof of Theorem 2.2 that $\mathfrak{T}^{\alpha}(E)$ has Property $S_{\sigma}$ if and only if $\mathfrak{N}^{\alpha}(\gamma)$ has Property $S_{\sigma}$ for all $\gamma$ in $E$. In particular, if $\mathscr{T}^{\alpha}(\gamma)$ has Property $S_{\sigma}$ for all $\gamma$ in $\Gamma$, then $\mathfrak{K}=\mathfrak{K}^{\alpha}(\Gamma)$ has Property $S_{\sigma}$.

Proposition 2.4. Let $(\mathfrak{T}, G, \alpha)$ be a $W^{*}$-dynamical system, and suppose that $G$ is compact or discrete. Then the crossed product $\Re(\Re, G, \alpha)$ has Property $S_{\sigma}$ if and only if $\Re$ has Property $S_{\sigma}$.

Proof. Let $\hat{\alpha}$ be the dual action of $\Gamma=\hat{G}$ on $\Re(\Re, G, \alpha)$ [20], let $\Re_{0}=$ $\Re(\Re, G, \alpha)$, and $\Re=\Re\left(\Re_{0}, \Gamma, \hat{\alpha}\right)$. Then by Takesaki's duality theorem [20, Theorem 4.5],

$$
\Re \cong \Re \bar{\otimes} B\left(L^{2}(G)\right)
$$

Since Property $S_{\sigma}$ is preserved by *-isomorphisms, it follows from Proposition 1.15 and Theorem 1.9 that $\mathfrak{\pi}$ has Property $S_{\sigma}$ if and only if $\Re$ does.

Now suppose $G$ is discrete. Let $\left\{\pi_{\alpha}, \lambda\right\}$ be the covariant representation of ( $\Re, G, \alpha)$ given by (3.1) in [20]. Then for each $g \in G,\left(\mathscr{N}_{0}\right)^{\hat{\alpha}}(g)=\pi_{\alpha}(\mathfrak{R}) \lambda(g)$ [16, Corollary 4.3.2]. Moreover, it is easily verified that if $\mathcal{S}$ is a $\sigma$-weakly closed subspace of $B(\mathcal{H})$ for some Hilbert space $\mathcal{H}$, and $a$ is an invertible operator in $B(\mathcal{H})$, then $\mathcal{S}$ has Property $S_{\sigma}$ if and only if $\mathcal{S} a$ has Property $S_{\sigma}$. Hence $\pi_{\alpha}(\mathfrak{T})$ has Property $S_{\sigma}$ if and only if $\left(\Re_{0}\right)^{\hat{\alpha}}(g)$ has Property $S_{\sigma}$ for all $g \in G$. Combining this fact with Remark 2.3, we see that $\pi_{\alpha}(\mathfrak{R})$ has Property $S_{\sigma}$ if and only if $\mathfrak{K}_{0}$ has Property $S_{\sigma}$. But $\pi_{\alpha}$ is a faithful normal representation, so $\Re$ has Property $S_{\sigma}$ if and only if $\pi_{\alpha}(\Re)$ does. This completes the proof in the discrete case.

Next suppose $G$ is compact. Then $\Gamma$ is discrete, and so by what we have just shown, $\Re_{0}$ has Property $S_{\sigma}$ if and only if $\Re$ does. But, as noted above, $\Re$ has Property $S_{\sigma}$ if and only if $\mathfrak{K}$ does, and so $\mathfrak{K}_{0}$ has Property $S_{\sigma}$ if and only if $\mathfrak{K}$ has Property $S_{\sigma}$.

TheOrem 2.5. Let $\left(\Re, G_{1}, \alpha\right)$ and $\left(\Re, G_{2}, \beta\right)$ be $W^{*}$-dynamical systems, and suppose $G_{1}$ is compact, and $\mathfrak{N}$ has Property $S_{\sigma}$. Then (2.1) holds for all $E_{1} \subset \Gamma_{1}$ and $E_{2} \subset \Gamma_{2}$.

Proof. It follows from Theorem 2.2 and Remark 1.5 that $F\left(\Re^{\alpha}\left(E_{1}\right), \Re^{\beta}\left(E_{2}\right)\right)=$ $\Re^{\alpha}\left(E_{1}\right) \bar{\otimes} \Re^{\beta}\left(E_{2}\right)$. Combining this fact with (2.2) and Proposition 2.1, we get (2.1).

Note that the proof of Theorem 2.5 shows that $(2.1)$ holds whenever $\mathfrak{M}^{\alpha}\left(E_{1}\right)$ has Property $S_{\sigma}$. Thus it would be of great interest to know whether Theorem 2.2 remains valid if we delete the hypothesis that $G$ be compact. If $G$ is not compact, the projections onto the spectral eigenspaces are no longer available, and the method of proof of Theorem 2.2 does not generalize. However, there is a partial substitute for these projections. Let $(\Re, G, \alpha)$ be a $W^{*}$-dynamical system. Suppose $E \subset \Gamma$ is 
open, and $K \subset E$ is compact. Then we can choose an open set $V$ with compact closure such that $K \subset V \subset \bar{V} \subset E$, and an $f \in L^{1}(G)$ such that $\hat{f}=1$ on $\bar{V}$ and supp $\hat{f} \subset E$. Then $\alpha(f)$ is a $\sigma$-weakly continuous linear map from $\mathcal{N}$ into $\mathcal{M}^{\alpha}(E)$ $[5,2.1 .3(\mathrm{~g})]$. Moreover, if $x \in \mathcal{O} \mathbb{R}^{\alpha}(K)$, then $\hat{f}=1$ on a neighborhood of $\operatorname{sp}_{\alpha}(x)$, and so $\alpha(f)(x)=x[5,2.1 .3(\mathrm{k})]$. If (i) $\alpha(f)$ is the identity on $\mathcal{O K}^{\alpha}(E)$ or (ii) the range of $\alpha(f)$ is $\mathfrak{N}^{\alpha}(K)$, then we can use Proposition 1.19 to show that (i) $\mathfrak{N}^{\alpha}(E)$ or (ii) $\mathfrak{T}^{\alpha}(K)$ has Property $S_{\sigma}$. However, it is not hard to show (using [5, 2.1.3(h)] and the regularity of $L^{1}(G)$ ) that (i) occurs only if $\hat{f}=1$ on $E \cap \operatorname{sp} \alpha$ (where $\operatorname{sp} \alpha=\{\gamma \in \Gamma$ : $\hat{f}(\gamma)=0$ whenever $\alpha(f)=0\}$ ), while (ii) occurs only if $K \cap \operatorname{sp} \alpha=\{\gamma: \hat{f}(\gamma) \neq 0\} \cap$ $\operatorname{sp} \alpha$. In the first case $E \cap \operatorname{sp} \alpha=\{\gamma: \hat{f}(\gamma)=1\} \cap \operatorname{sp} \alpha$ is closed, while in the second case $K \cap \operatorname{sp} \alpha$ is relatively open in $\operatorname{sp} \alpha$. (These two special cases are treated in Corollary 2.7 below.) In particular, if $E \cap \operatorname{sp} \alpha$ is not closed, we cannot apply Proposition 1.19 to $\mathfrak{O K}^{\alpha}(E)$. However, we can prove a weaker version of Theorem 2.2, which is strong enough to imply that (2.1) holds when $\mathscr{N}$ has Property $S_{\sigma}$ and $E_{1}$ is open.

TheOREM 2.6. Let (으, $G, \alpha$ ) be a $W^{*}$-dynamical system, and suppose ON has Property $S_{\sigma}, E \subset \Gamma$ is open, and $K \subset E$ is compact. If $T$ is any $\sigma$-weakly closed subspace of a von Neumann algebra $\mathcal{N}$, then

$$
F\left(\mathscr{N}^{\alpha}(K), \mathscr{T}\right) \subset \mathscr{N}^{\alpha}(E) \bar{\otimes} \mathscr{T}
$$

Proof. Let $R=\mathscr{N} \bar{\otimes} \mathscr{X}$, and define a $W^{*}$-dynamical system $(\mathscr{R}, G, \beta)$ by setting $\beta_{g}=\alpha_{g} \otimes 1$, where 1 is the identity automorphism of $\Re$. Then

$$
L_{\psi}(\beta(f)(x))=\alpha(f)\left(L_{\psi}(x)\right) \quad\left(x \in \mathcal{R}, f \in L^{1}(G), \psi \in \mathscr{K}_{*}\right) .
$$

Hence if $F$ is a closed subset of $\Gamma$, and $x \in R$, then $x \in R^{\beta}(F)$ if and only if $\beta(f)(x)=0$ for all $f \in I_{0}(F)\left[1\right.$, p. 225] if and only if $\alpha(f)\left(L_{\psi}(x)\right)=0$ for all $f \in I_{0}(F)$ and all $\psi \in \mathfrak{N}_{*}$ if and only if $L_{\psi}(x) \in \mathfrak{N}^{\alpha}(F)$ for all $\psi \in \mathfrak{T}_{*}$. Thus

$$
\Re^{\beta}(F)=F\left(\Re^{\alpha}(F), \Re\right)
$$

for any closed subset $F$ of $\Gamma$. Choose $f \in L^{1}(G)$ such that $\hat{f}=1$ on a neighborhood of $K$, and $\operatorname{supp} \hat{f} \subset E$. Then, since $\beta(f)(a \otimes b)=\alpha(f)(a) \otimes b(a \in \Re, b \in \Re)$, $\beta(f)(\mathscr{N} \bar{\otimes} \mathcal{T}) \subseteq \Re^{\alpha}(E) \bar{\otimes} \mathcal{T}$. But $F\left(\Re^{\alpha}(K), \mathcal{T}\right) \subset F(\Re, \mathcal{T})=\mathscr{\Re} \bar{\otimes} \mathcal{T}$, and $\beta(f)(x)=x$ for $x \in \mathscr{R}^{\beta}(K)=F\left(\mathscr{T}^{\alpha}(K), \mathfrak{\Re}\right)$, so (2.10) holds.

COROllaRy 2.7. Let $(\mathfrak{N}, G, \alpha)$ be a $W^{*}$-dynamical system, and suppose $\mathfrak{N}$ has Property $S_{\sigma}$. Let $E \subset \Gamma$ be such that $\operatorname{sp} \alpha \cap E$ is relatively open and closed in $\operatorname{sp} \alpha$. Then $\Re^{\alpha}(E)$ has Property $S_{\sigma}$.

Proof. Let $\mathcal{T}$ be a $\sigma$-weakly closed subspace of a von Neumann algebra $\Re$, let $\Re=\Re \bar{\otimes} \Re$, and define $\beta$ as in the proof of Theorem 2.6. Let $F=\operatorname{sp} \alpha \cap E$. Then $F$ is closed, there is an open subset $V$ of $\Gamma$ such that $\operatorname{sp} \alpha \cap V=F$, and $\Re^{\alpha}(E)=$ $\Re^{\alpha}(F)=\Re^{\alpha}(V)$. Let $x \in F\left(\Re^{\alpha}(E), \mathcal{T}\right)$, let $f \in L^{1}(G)$ be such that supp $\hat{f}$ is compact, and set $K=\operatorname{sp}_{\beta}(\beta(f)(x))$. Then $K \subset \operatorname{supp} \hat{f} \cap \operatorname{sp}_{\beta}(x)$ [5, 2.1.3(g)]. Hence $K$ is compact, and $K \subset F \subset V$ by (2.11). Moreover, $R_{\varphi}(\beta(f)(x))=R_{\varphi \circ \alpha(f)}(x) \in \mathcal{T}$ for all $\varphi \in \mathscr{N}_{*}$, and $\beta(f)(x) \in \mathscr{R}^{\beta}(K)=F\left(\mathscr{T}^{\alpha}(K), \mathfrak{N}\right)$, so $\beta(f)(x) \in$ $F\left(\Re^{\alpha}(K), \mathcal{T}\right)$. Hence by Theorem 2.6, $\beta(f)(x) \in \Re^{\alpha}(V) \bar{\otimes} \mathcal{T}=\Re^{\alpha}(E) \bar{\otimes} \mathcal{T}$. 
Since $x$ is in the $\sigma$-weak closure of $\left\{\beta(f)(x): f \in L^{1}(G),\|f\|_{1} \leqslant 1\right.$ and supp $\hat{f}$ is compact\} $[5,2.1 .4]$, we conclude that $x \in \mathcal{M}^{\alpha}(E) \bar{\otimes} \mathcal{T}$, and hence $\mathscr{M}^{\alpha}(E)$ has Property $S_{\sigma}$.

THEOREM 2.8. Let (OM, $\left.G_{1}, \alpha\right)$ and $\left(\mathcal{N}, G_{2}, \beta\right)$ be $W^{*}$-dynamical systems and suppose that ơ has Property $S_{\sigma}$. If $E_{1} \subset \Gamma_{1}$ is open, and $E_{2} \subset \Gamma_{2}$ is arbitrary, then (2.1) holds.

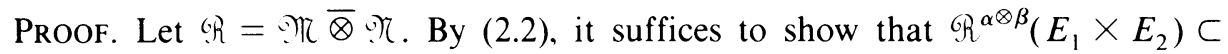
$\because \eta^{\alpha}\left(E_{1}\right) \bar{\otimes} \Re^{\beta}\left(E_{2}\right)$. Since $\cap \eta^{\alpha}\left(E_{1}\right) \bar{\otimes} \Re^{\beta}\left(E_{2}\right)$ is a $\sigma$-weakly closed subspace, it suffices to show that if $\operatorname{sp}_{\alpha \otimes \beta}(x) \subset E_{1} \times E_{2}$, then $x \in \Re^{\alpha}\left(E_{1}\right) \bar{\otimes} \Re^{\beta}\left(E_{2}\right)$, and by $\left[5,2.1 .3(\mathrm{~g})\right.$ and 2.1.4], we may assume $\operatorname{sp}_{\alpha \otimes \beta}(x)$ is compact. Let $K=\operatorname{sp}_{\alpha \otimes \beta}(x)$, and let $K_{1}$ be the projection of $K$ on $\Gamma_{1}$. Then $K_{1} \subset E_{1}$ is compact, and $K \subset K_{1} \times E_{2}$. Hence, by Proposition 2.1 and Theorem 2.6,

$$
x \in \Re^{\alpha \otimes \beta}\left(K_{1} \times E_{2}\right) \subset F\left(\Re^{\alpha}\left(K_{1}\right), \Re^{\beta}\left(E_{2}\right)\right) \subset \Re^{\alpha}\left(E_{1}\right) \bar{\otimes} \Re^{\beta}\left(E_{2}\right),
$$

as required.

If both $E_{1}$ and $E_{2}$ are open, we can show that (2.1) holds without the requirement that $)$ have Property $S_{\sigma}$. Although the proof of this fact does not use slice maps, we include it here for completeness.

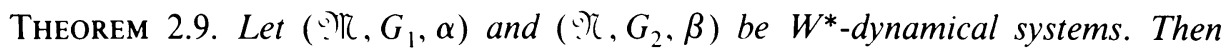
(2.1) holds whenever both $E_{1}$ and $E_{2}$ are open sets.

Proof. Let $\Re=\Re \bar{\otimes} \Re$, let $G=G_{1} \times G_{2}$, and $\Gamma=\Gamma_{1} \times \Gamma_{2}$. By (2.2), it suffices to show that $?^{\alpha \otimes \beta}\left(E_{1} \times E_{2}\right) \subset \Re^{\alpha}\left(E_{1}\right) \otimes \Re^{\beta}\left(E_{2}\right)$. Since $E_{1} \times E_{2}$ is open, ( $R{ }^{\alpha \otimes \beta}\left(E_{1} \times E_{2}\right)$ is the $\sigma$-weak closure of $\left\{(\alpha \otimes \beta)(f)(x): x \in \mathscr{R}, f \in L^{1}(G)\right.$, and supp $\left.\hat{f} \subset E_{1} \times E_{2}\right\} \quad[17,2.3 .3(i i)]$. Note that $\left\{f \in L^{1}(G)\right.$ : $\left.\operatorname{supp} \hat{f} \subset E_{1} \times E_{2}\right\}=$ $I_{0}\left(\Gamma \backslash\left(E_{1} \times E_{2}\right)\right)$. Let $J$ be the closed subspace of $L^{1}(G)$ generated by $\left\{f_{1} \otimes f_{2}\right.$ : $\left.f_{i} \in I_{0}\left(\Gamma_{i} \backslash E_{i}\right), i=1,2\right\}$. By arguments similar to those in the second part of the proof of Proposition 2.1, $J$ is a closed ideal, and $Z(J)=\Gamma \backslash\left(E_{1} \times E_{2}\right)$. Hence $I_{0}\left(\Gamma \backslash\left(E_{1} \times E_{2}\right)\right) \subset J$. Thus, it suffices to show that if $f_{i} \in I_{0}\left(\Gamma_{i} \backslash E_{i}\right), i=1,2$, then

$$
(\alpha \otimes \beta)\left(f_{1} \otimes f_{2}\right)(a \otimes b) \in \mathfrak{N}^{\alpha}\left(E_{1}\right) \otimes \mathscr{N}^{\beta}\left(E_{2}\right) \quad(a \in \mathfrak{N}, b \in \mathfrak{N}) \text {. }
$$

But $I_{0}\left(\Gamma_{i} \backslash E_{i}\right)=\left\{f \in L^{\mathrm{l}}\left(G_{i}\right)\right.$ : $\left.\operatorname{supp} \hat{f} \subset E_{i}\right\}, i=1,2$, and

$$
(\alpha \otimes \beta)\left(f_{1} \otimes f_{2}\right)(a \otimes b)=\alpha\left(f_{1}\right)(a) \otimes \beta\left(f_{2}\right)(b) \quad(a \in \mathfrak{N}, b \in \mathfrak{K}),
$$

so (2.12) follows from [17, 2.3.3(ii)].

3. Applications to subspace lattices and reflexive algebras. In this section, we will always assume that Hilbert spaces are separable. A subspace lattice $\mathcal{L}$ in $B(\mathcal{H})$ is a collection of (selfadjoint) projections on $\mathcal{H}$ closed under the lattice operations $\wedge$ (intersection) and $\vee$ (closed linear span) (where we identify projections with their ranges), which contains 0 and 1 , and which is closed in the strong operator topology. If the elements of $\varrho$ pairwise commute, $\mathcal{L}$ is a commutative subspace lattice (or CSL). If $\mathcal{L}$ is a subspace lattice, alg $\mathcal{L}=\{x \in B(\mathcal{H}): x p=\operatorname{pxp}$ for all $p \in \mathcal{L}\}$ is a $\sigma$-weakly closed subalgebra of $B(\mathcal{H})$. If $S$ is any subset of $B(\mathcal{H})$, then lat $S$, the set of projections on $\mathcal{H}$ left invariant by the elements of $S$, is a subspace lattice. A subspace 
lattice $E^{2}$ [resp. $S \subset B(\tilde{H})$ ] is reflexive if lat alg $E=E[$ resp. alg lat $S=S$ ]. Commutative subspace lattices were first studied systematically by Arveson [2], who developed powerful techniques for studying these lattices and their associated reflexive algebras. We refer the reader to [2] for a detailed treatment of CSL's.

If $\mathcal{O} \subset B(\mathcal{H})$ is a von Neumann algebra, and $E \subset \Re$ is a subspace lattice, we write $\Re$-alg $E$ for $($ alg $E) \cap \mathcal{O}$. If $E_{1} \subset \Re$ and $E_{2} \subset \Re$ are subspace lattices, we can ask: when do we have

$$
\Re \text {-algE }=\left(\Re \text {-alg } E_{1}\right) \bar{\otimes}\left(\Re-a l g E_{2}\right) \text {, }
$$

where $\mathscr{R}=\mathscr{N} \bar{\otimes} \mathcal{K}$, and $E=E_{1} \otimes E_{2}$ is the subspace lattice generated by $\left\{p_{1} \otimes p_{2}\right.$ : $\left.p_{i} \in \mathcal{L}_{i}\right\}$ ? If $\mathfrak{N}=B\left(\mathcal{H}_{1}\right)$ and $\mathcal{K}=B\left(\mathcal{K}_{2}\right)$, (3.1) becomes, simply,

$$
\operatorname{alg} E=\left(\operatorname{alg} E_{1}\right) \bar{\otimes}\left(\operatorname{alg} \varrho_{2}\right) \text {. }
$$

Note that if (3.2) is always valid, then the tensor product of reflexive algebras is always reflexive. Moreover, if $E_{1} \subset \mathcal{N}$ and $L_{2} \subset \Re$ are the projection lattices of von Neumann subalgebras $\mathcal{N}_{1}$ and $\mathcal{N}_{1}$, then (3.1) becomes

$$
\left(\Re_{1} \bar{\otimes} \Re_{1}\right)^{\prime} \cap(\Re \bar{\otimes} \Re)=\left(\Re_{1}^{\prime} \cap \Re\right) \bar{\Re}\left(\Re_{1}^{\prime} \cap \Re\right) \text {. }
$$

It follows from (1.11), (1.8) and (1.7) that this relative commutant version of Tomita's theorem is always valid (see also [24, p. 167]). Gilfeather, Hopenwasser and Larson showed in [10, Theorem 2.6] that (3.2) holds if $L_{1}$ and $L_{2}$ are chains (totally ordered CSL's). Hopenwasser, Laurie and Moore have shown that, more generally, (3.2) is valid for completely distributive CSL's [14], and K. Harrison has proved (3.2) in the case when $L_{1}$ is a chain and $\mathcal{L}_{2}$ is a finite width CSL [13]. The author has shown [15] that (3.1) holds if $\varrho_{1}$ and $\varrho_{2}$ are CSL's with totally atomic cores. (The core of $\mathcal{L}$ is the von Neumann algebra $\mathcal{L}^{\prime \prime}$ generated by $\mathcal{L}$.)

If $\mathscr{L}_{1} \subset \mathscr{N}$ and $\mathscr{L}_{2} \subset \mathcal{K}$ are subspace lattices, and $x \in \mathcal{R}$-alg $\mathscr{L}$, then if $e \in \mathscr{L}_{2}$ and $\varphi \in \mathfrak{K}_{*}$,

$$
e R_{\varphi}(x) e=R_{\varphi}((1 \otimes e) x(1 \otimes e))=R_{\varphi}(x(1 \otimes e))=R_{\varphi}(x) e,
$$

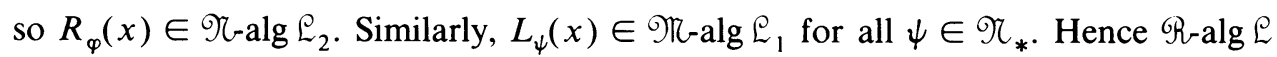
$\subset F\left(\Re\right.$-alg $\left.\varrho_{1}, \Re_{\text {-alg }} \varrho_{2}\right)$. Another use of (1.3) and (1.4) proves the reverse inclusion, and we conclude:

$$
\Re \text {-alg } \varrho=F\left(\text { T -alg } \varrho_{1}, \Re_{\text {-alg }} \varrho_{2}\right) \text {. }
$$

Hence if (1.10) is always valid, then (3.1) is always valid. It also follows from (3.3) (and Remark 1.5) that if $\mathscr{N}-$-alg $\mathcal{L}_{1}$ has Property $S_{\sigma}$, then (3.1) holds for any subspace lattice $\mathcal{L}_{2} \subset \mathcal{K}$. In particular, an application of Proposition 1.7 yields the following result.

Proposition 3.1. Let $\mathcal{L}_{1} \subset \Re$ and $\varrho_{2} \subset \Re$ be subspace lattices, and suppose that $\mathfrak{T}$ is finite-dimensional. Then (3.1) holds.

For certain CSL's, there is a connection between (3.1) and (2.1), established in [15]. For the convenience of the reader, we will state the appropriate definitions and results from [15]. If $G$ is a locally compact abelian group, a positive semigroup 
$\Sigma \subset \Gamma$ is a subsemigroup of $\Gamma$ also satisfying $\Sigma \cap(-\Sigma)=\{0\}$ and $\Sigma=\overline{\text { int } \Sigma}$. A CSL $\Sigma \subset \mathcal{O}$ is $(G, \Sigma)$-analytic if there is a strongly continuous unitary representation $u$ of $G$ on the Hilbert space $\mathcal{H}$ of $\mathscr{N}$ such that $u_{g} \in \mathscr{N}$ for all $g \in G$ and $\mathcal{L}$ is the subspace lattice generated by $\{e(\gamma+\Sigma): \gamma \in \Gamma\}$, where $e(\cdot)$ is the projection-valued measure on $\Gamma$ associated with $u$ by Stone's Theorem. If $\alpha=a d u$ (i.e. $\alpha_{g}(x)=u_{g} x u_{g}^{*}$, $x \in \mathfrak{N}, g \in G)$, then $\Re$-alg $\mathscr{L}=\mathfrak{O}^{\alpha}(\Sigma)$. (The algebras $\mathfrak{M}^{\alpha}(\Sigma), \Sigma$ a positive semigroup, are called algebras of analytic operators, and were first studied by Loebl and Muhly [16]. Note that if $G=\mathbf{R}$, and $\Sigma=[0, \infty)$, then $x \in \mathcal{N R}^{\alpha}(\Sigma)$ if and only if all of the maps $g \rightarrow\left\langle\alpha_{g}(x), \varphi\right\rangle\left(\varphi \in \mathfrak{M} \mathbb{R}_{*}\right)$ are in $H^{\infty}(\mathbf{R})$.) If $\varrho_{1} \subset \mathfrak{M}, \varrho_{2} \subset \mathfrak{K}$, and $E_{i}$ is $\left(G_{i}, \Sigma_{i}\right)$-analytic, $i=1,2, E=E_{1} \otimes \mathcal{L}_{2}$ is $\left(G_{1} \times G_{2}, \Sigma_{1} \times \Sigma_{2}\right)$-analytic, and $\left(R^{\alpha \otimes \beta}\left(\Sigma_{1} \times \Sigma_{2}\right)=(R\right.$-alg $E$, where $(R=) \mathbb{R} \otimes$. Hence, in the case of analytic CSL's, (3.1) is a special case of (2.1), and the results of $\$ 2$ can be applied.

THEOREM 3.2. Let $E_{1} \subset \mathfrak{K}$ and $E_{2} \subset \Re$ be subspace lattices, and suppose $\mathbb{N}$ has Property $S_{\sigma}$ and $E_{1}$ is a CSL with totally atomic core. Then (3.1) holds.

Proof. By [15, Theorem 4.4], $E_{1}$ is $(G, \Sigma)$-analytic for some compact group $G$, and

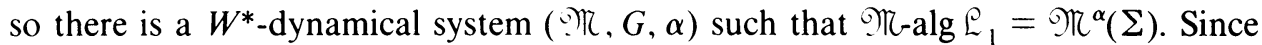
$G$ is compact, and $) \pi$ has Property $S_{\sigma}$, it follows from Theorem 2.2 that $\Re$-alg $\mathcal{L}_{1}$ has Property $S_{\sigma}$, and so (3.1) follows immediately from (3.3).

Another important class of analytic CSL's is the class of finite width CSL's. (A CSL has finite width if it is generated by a finite number of commuting chains.) In

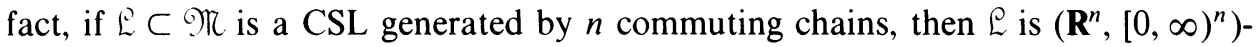
analytic, and, conversely, if $\left(\Re, \mathbf{R}^{n}, \alpha\right)$ is an inner $W^{*}$-dynamical system, then there

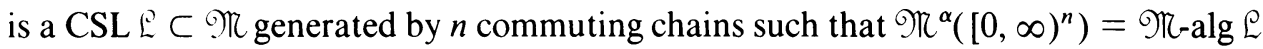
[15, Theorem 2.6]. (The case $n=1$ was proved by Loebl and Muhly [16, Theorem 4.2.3].) In particular, since (3.2) holds for chains and every one-parameter dynamical system on $B(\mathscr{H})$ is inner, $(2.1)$ holds if $\mathscr{N}=B\left(\mathcal{H}_{1}\right), \mathscr{N}=B\left(\mathcal{H}_{2}\right), G_{1}=G_{2}=\mathbf{R}$, and $E_{1}=E_{2}=[0, \infty)$. We will next give a direct proof of a more general result, using a theorem of Zsidó.

Recall that if $G$ is a locally compact abelian group, a set $E \subset \Gamma$ is polyhedral if there exist closed subsets $E_{1}, \ldots, E_{n}$ of $\Gamma$ such that $E_{i} \cap\left(-E_{i}\right)$ and $E_{i} \cup\left(-E_{i}\right)$, $1 \leqslant i \leqslant n$, are subgroups of $\Gamma$, and $E$ belongs to the translation-invariant ring of subsets of $\Gamma$ generated by $\left\{E_{1}, \ldots, E_{n}\right\}$. Note that $[0, \infty)^{n}$ is polyhedral in $\mathbf{R}^{n}$ for all $n$.

THEOREM 3.3. Let ( $\Re, \mathbf{R}, \alpha)$ and $(\Re, G, \beta)$ be $W^{*}$-dynamical systems, and suppose T and $\mathfrak{T}^{\alpha}(0)$ have Property $S_{\sigma}$. Let $E_{1}=[0, \infty)$. Then (2.1) holds for any polyhedral set $E_{2}$.

Proof. Let $\Re=\Re \bar{\otimes} \Re$. Since $E_{2}$ is polyhedral in $\Gamma_{2},\{0\} \times E_{2}$ is polyhedral in $\Gamma=\Gamma_{1} \times \Gamma_{2}$. Hence by [29, Theorem 3.7 and Lemma 3.8],

$$
\Re^{\alpha \otimes \beta}\left(E_{1} \times E_{2}\right)=\Re^{\alpha \otimes \beta}\left(\left(\{0\} \times E_{2}\right) \cup\left((0, \infty) \times E_{2}\right)\right)
$$

is the $\sigma$-weakly closed linear span of $\Re^{\alpha \otimes \beta}\left(\{0\} \times E_{2}\right)$ and $\Re^{\alpha \otimes \beta}\left((0, \infty) \times E_{2}\right)$. Since $\mathfrak{T}^{\alpha}(0)$ has Property $S_{\sigma}, \quad \Re^{\alpha \otimes \beta}\left(\{0\} \times E_{2}\right)=\mathfrak{M}^{\alpha}(0) \bar{\otimes} \Re^{\beta}\left(E_{2}\right) \subset \mathfrak{M}^{\alpha}\left(E_{1}\right) \bar{\otimes}$ $\Re^{\beta}\left(E_{2}\right)$. Since $(0, \infty)$ is open, and $\Re$ has Property $S_{\sigma}$, it follows from Theorem 2.8 
that $\Re^{\alpha \otimes \beta}\left((0, \infty) \times E_{2}\right)=\Re^{\alpha}\left((0, \infty) \bar{\otimes} \Re^{\beta}\left(E_{2}\right) \subset \Re^{\alpha}\left(E_{1}\right) \bar{\otimes} \Re^{\beta}\left(E_{2}\right)\right.$. Hence $\Re^{\alpha \otimes \beta}\left(E_{1} \times E_{2}\right) \subset \mathcal{N}^{\alpha}\left(E_{1}\right) \bar{\otimes} \mathcal{N}^{\beta}\left(E_{2}\right)$. But the reverse inclusion always holds, and so we get $(2.1)$.

Corollary 3.4. Let ( O $\mathrm{M}, \mathbf{R}, \alpha)$ and $\left(\Im, \mathbf{R}^{n}, \beta\right)$ be $W^{*}$-dynamical systems and suppose $\mathfrak{⿰}$ and $\mathfrak{M}^{\alpha}(0)$ have Property $S_{\sigma}$. Then

$$
(\Re \bar{\otimes} \Re)^{\alpha \otimes \beta}\left([0, \infty) \times[0, \infty)^{n}\right)=\Re^{\alpha}([0, \infty)) \bar{\otimes} \Re^{\beta}\left([0, \infty)^{n}\right) .
$$

COROLlaRY 3.5. Let $E_{1} \subset \mathcal{M}$ be a chain, and $E_{2} \subset \Re$ be a finite width CSL. Suppose that $\mathfrak{M}$ and $E_{1}^{\prime} \cap \mathcal{M}$ have Property $S_{\sigma}$. Then (3.1) holds.

Proof. Suppose $E_{2}$ is generated by $n$ commuting chains. Then by [15, Theorems 2.6 and 4.1 and Proposition 2.4] we can choose inner $W^{*}$-dynamical systems $(\mathscr{N}, \mathbf{R}, \alpha)$ and $\left(\Re, \mathbf{R}^{n}, \beta\right)$ such that $\Re$-alg $\mathcal{L}_{1}=\mathscr{N}^{\alpha}([0, \infty))$, $)$-alg $\mathscr{L}_{2}=$ $\Re^{\beta}\left([0, \infty)^{n}\right), \mathcal{R}$-alg $\mathcal{L}=\mathscr{G}^{\alpha}{ }^{\otimes \beta}\left([0, \infty) \times[0, \infty)^{n}\right)$, and $\mathfrak{M}^{\alpha}(0)=\mathcal{L}_{1}^{\prime} \cap$ ? $)$. Now apply Corollary 3.4.

If $\Re=B(\mathcal{H})$ in Corollary 3.5 , then both $\Re$ and $\mathscr{L}_{1}^{\prime} \cap \Re=\left(\mathcal{L}_{1}^{\prime \prime}\right)^{\prime}$ are type I, and hence have Property $S_{\sigma}$. Thus Corollary 3.5 generalizes Harrison's result that (3.2) holds when $\mathcal{L}_{1}$ is a chain and $\mathcal{L}_{2}$ is a finite width CSL [13].

We conclude this section with an application of some of the above results to synthetic CSL's. Recall from [2] that an operator algebra $A \subset B(\mathcal{H})$ is prereflexive if $A \cap A^{*}=(\text { lat } A)^{\prime}$, and that if $\varrho$ is a CSL, and $\circlearrowright$ is the class of all $\sigma$-weakly closed prereflexive algebras $Q$ satisfying lat $\mathcal{Q}=\mathcal{L}$, then $\mathcal{Q}$ has a smallest element (denoted $\left.Q_{\text {min }}(\mathcal{L})\right)$ and a largest element, alg $E[2,2.1 .8]$. If $Q_{\text {min }}(E)=$ alg $E$, then $L^{\prime}$ is said to be synthetic. The name synthetic comes from an analogy with $S$-sets (sets of spectral synthesis) in harmonic analysis, which is discussed in detail in $[2, \S 2.2]$. Theorem 2.2.11 in [2] and Proposition 2.1 in [10] suggest that the proper analogue of the tensor product $\varrho_{1} \otimes L_{2}$ of synthetic CSL's $\varrho_{1}$ and $L_{2}$ is the product $E_{1} \times E_{2}$ of $S$-sets $E_{1}$ and $E_{2}$. If $E_{1}$ and $E_{2}$ are $S$-sets, and if either $A\left(E_{1}\right)$ or $A\left(E_{2}\right)[25, \S 4.1]$ has the approximation property [11], then $E_{1} \times E_{2}$ is an $S$-set. (Combine [25, Theorem 1.51] with [11, Proposition 3] and [22, Theorem 4].) This suggests that the tensor product of synthetic CSL's is synthetic. We will sinow that this is true in the special case when one of the CSL's has totally atomic core.

If $\mathcal{L}_{1}$ and $\mathcal{L}_{2}$ are CSL's, then by Arveson's spectral theorem for CSL's [2, 1.3.1], for each $i, i=1,2$, there is a compact metric space $X_{i}$, a finite Borel measure $m_{i}$ on $X_{i}$, and a closed partial order $\leqslant_{i}$ on $X_{i}$, such that $\mathcal{L}_{i}$ is unitarily equivalent to $\mathcal{L}\left(X_{i}, \leqslant_{i}, m_{i}\right)=\left\{P_{E}: E\right.$ is an increasing Borel subset of $\left.X_{i}\right\}$, where $P_{E}$ is the operator on $L^{2}\left(X_{i}, m_{i}\right)$ corresponding to multiplication by the characteristic function of $E$. Since the property of being synthetic is preserved under unitary equivalence, in what follows we may assume $\mathcal{L}_{i}=\mathcal{L}\left(X_{i}, \leqslant_{i}, m_{i}\right)$. In this case, $\varrho_{1} \otimes \varrho_{2}=\ell(X, \leqslant, m)$, where $X=X_{1} \times X_{2}, m=m_{1} \times m_{2}$ is the product measure, and $\leqslant$ is the product partial order (i.e. $\left(x_{1}, x_{2}\right) \leqslant\left(y_{1}, y_{2}\right)$ if and only if $x_{i} \leqslant y_{i}$ for $\left.i=1,2\right)$ [10, Proposition 2.1]. Moreover, for $i=1,2, Q_{\text {min }}\left(\varrho_{i}\right)$ is the $\sigma$-weak closure of the set of pseudo-integral operators on $L^{2}\left(X_{i}, m_{i}\right)$ corresponding to the measures in $A\left(G_{i}, m_{i}\right)$, where $G_{i}$ is the graph of $\leqslant_{i}\left[2, \S \S 1.5\right.$ and 1.6]. It is easily checked that if $s_{i}$ is a 
pseudo-integral operator corresponding to a measure in $A\left(G_{i}, m_{i}\right), i=1,2$, then $s_{1} \otimes s_{2}$ is a pseudo-integral operator corresponding to a measure in $A(G, m)$, where $G$ is the graph of $\leqslant$, and so $s_{1} \otimes s_{2} \in \mathcal{C H}_{\text {min }}^{\prime}\left(E_{1}^{\prime} \otimes L_{2}\right)$. Hence

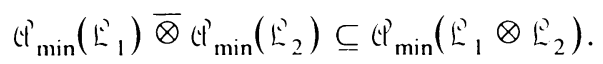

THEOREM 3.6. Let $\mathscr{L}_{1}$ and $\mathscr{L}_{2}$ be synthetic CSL's and suppose $E_{1}^{\prime \prime}$ is totally atomic. Then $\mathbb{I}_{1} \otimes I_{2}$ is synthetic.

Proof. Since $L_{1}$, has totally atomic core, and $B(\mathcal{H})$ has Property $S_{\sigma}$, it follows from Theorem 3.2 that $\operatorname{alg}\left(E_{1} \otimes E_{2}\right)=\left(\operatorname{alg} E_{1}\right) \otimes\left(\operatorname{alg} E_{2}\right)$. Since $E_{i}$ is synthetic, $\operatorname{alg} E_{i}^{\prime}=C_{\min }^{\prime}\left(E_{i}^{\prime}\right), i=1,2$, and so $\operatorname{alg}\left(E_{1} \otimes E_{2}\right) \subseteq \mathcal{Q}_{\min }\left(E_{1} \otimes E_{2}\right)$, by (3.4). But the reverse inclusion always holds, so $E_{1} \otimes E_{2}$ is synthetic.

REMARK 3.7. It should be noted that if $E_{1}$ is any CSL with totally atomic core, then $L_{1}$ is synthetic, and so satisfies the hypotheses of Theorem 3.6. One way of seeing this is the following: If $E$ is a CSL with totally atomic core, then it follows from [15. Theorems 4.4 and 3.3] that there is a countable discrete metric space $X$, a partial order $\leqslant$ on $X$, and a $\sigma$-finite Borel measure $m$ on $X$ such that $\mathcal{E}$ is unitarily equivalent to $E(X, \leqslant, m)$. Since $X \times X$ is discrete, the graph of $\leqslant$ is automatically synthetic relative to $m[2,2.2 .9$ (ii)]. Hence $\mathcal{L}(X, \leqslant, m)$ is a synthetic lattice $[2,2.2 .11]$, and so $L^{\prime}$ is synthetic. Alternately, one could note that $\mathcal{L}$ is a completely distributive CSL, and hence is synthetic by [14, Corollary 9].

\section{REFERENCES}

I. W. Arveson, On groups of automorphisms of operator algebras, J. Funct. Anal. 15 (1974), 217-243.

2. . Operator algebras and invariant subspaces, Ann. of Math. (2) 100 (1974), 433-532.

3. . The harmonic analysis of automorphism groups, Proc. Sympos. Pure Math., vol. 38. Amer. Math. Soc.. Providence, R. I., pp. 199-269.

4. M. Choi and E. Effros, Separable nuclear $C^{*}$-algebras and injectivity, Duke Math. J. 43 (1976). 309-322.

5. A. Connes, Une classification des facteurs de type III, Ann. Sci. École Norm. Sup. 6 (1973), 133-252.

6. Classification of injective factors, Ann. of Math. (2) 104 (1976), 73-116.

7. On the classification of von Neumann algebras and their automorphism, Sympos. Math. 20 (1976). 435-478.

8. J. De Cannière and U. Haagerup, Multipliers of the Fourier algebras of some simple Lie groups and their discrete subgroups, preprint.

9. E. Effros and C. Lance, Tensor products of operator algehras, Adv. in Math. 25 (1977), 1-34

10. F. (jilfeather, A. Hopenwasser and D. Larson, Reflexive algehras with finite width lattices: tensor products, cohomologl, compact perturhations, J. Funct. Anal. (to appear).

11. A. Cirothendieck, Produits tensoriels topologiques et espaces nucleaires. Mem. Amer. Math. Soc. No. $16(1955)$.

12. U. Haagerup, The reduced $C^{*}$-algehra of the free group on two generators, Proc. 18 th Scandinavian Congr. Math.. Progress in Math., vol. 11, Birkhäuser, Boston, Mass., 1981.

13. K. Harrison, Reflexivity and tensor-products for operator algebras and subspace lattices, preprint.

14. A. Hopenwasser, C. Lauric and R. Moore, Reflexive algehras with completely distributice suhspace lattices, preprint.

15. J. Kraus, $W^{*}$-dynamical systems and reflexive operator algehras, J. Operator Theory 8 (1982). $181-194$.

16. R. Locbl and P. Muhly, Analvticity and flows in von Neumann algehras. J. Funct. Anal. 29 (1978). $214-252$.

17. D. Olesen, On spectral subspaces and their applications to automorphism groups. Sympos. Math. 20 (1976), 253-296.

18. (j. Pedersen, $C^{*}$-algebras and their automorphism groups, Academic Press, London, 1979. 
19. W. Rudin, Fourier analysis on groups, Wiley, New York, 1962.

20. M. Takesaki, Duality in cross products and the structure of von Neumann algebras of type III, Acta Math. 131 (1973), 249-310.

21. Theory of operator algebras. I, Springer-Verlag, Berlin and New York, 1979.

22. J. Tomiyama, Tensor products of commutative Banach algebras, Tôhoku Math. J. 12 (1960), 147-154.

23. , Tensor products and projections of norm one in von Neumann algebras, Lecture Notes, University of Copenhagen, 1970.

24. __ Tensor products and approximation problems of $C^{*}$-algebras, Publ. Res. Inst. Math. Sci. 11 (1975), 163-183.

25. N. Varopoulos, Tensor algebras and harmonic analysis, Acta Math. 119 (1967), 51-112.

26. S. Wassermann, The slice map problem for $C^{*}$-algebras, Proc. London Math. Soc. (3) 32 (1976), 537-559.

27. __. On tensor products of certain group $C^{*}$-algebras, J. Funct. Anal. 23 (1976), 239-254.

28. __ A pathology in the ideal space of $L(H) \oslash L(H)$, Indiana Univ. Math. J. 27 (1978), 1011-1020.

29. L. Zsidó, On spectral subspaces associated to locally compact abelian groups of operators, Adv. in Math. 36 (1980), 213-276.

Department of Mathematics, State University of New York at Buffalo, Buffalo, New York 14214 